\title{
An order on circular permutations
}

\author{
Antoine Abram* Nathan Chapelier-Laget \\ Christophe Reutenauer* \\ Département de mathématiques \\ Université du Québec à Montréal \\ Québec, Canada \\ abram.antoine@courrier.uqam.ca nathan.chapelier@gmail.com \\ Reutenauer.Christophe@uqam.ca
}

Submitted: Oct 29, 2020; Accepted: May 17, 2021; Published: Jun 30, 2021

(C) The authors. Released under the CC BY-ND license (International 4.0).

\begin{abstract}
Motivated by the study of affine Weyl groups, a ranked poset structure is defined on the set of circular permutations in $S_{n}$ (that is, $n$-cycles). It is isomorphic to the poset of so-called admitted vectors, and to an interval in the affine symmetric group $\tilde{S}_{n}$ with the weak order. The poset is a semidistributive lattice, and the rank function, whose range is cubic in $n$, is computed by some special formula involving inversions. We prove also some links with Eulerian numbers, triangulations of an $n$-gon, and Young's lattice.
\end{abstract}

Mathematics Subject Classifications: 06A07, 05A05

\section{Contents}

1 Introduction 2

2 Ordering circular permutations 3

3 An isomorphism towards admitted vectors $\quad 8$

4 The functions $\delta$ and triangulations of an $n$-gon 19

5 Properties of the poset $\quad 21$

5.1 Lattice . . . . . . . . . . . . . . . . . . . . . . 21

5.2 Multiplicities in the Hasse diagram and Eulerian numbers . . . . . . . . . . 23

5.3 Limiting poset. . . . . . . . . . . . . . . . . 25

${ }^{*}$ Partially supported by an NSERC grant. 
$6 \quad$ An interval in the affine symmetric group $\quad 27$

6.1 Preliminaries on $\tilde{S}_{n} \ldots \ldots \ldots \ldots \ldots \ldots$

6.2 A poset isomorphism . . . . . . . . . . . . . . . . . 29

$\begin{array}{lll}7 & \text { Circular line diagrams } & 35\end{array}$

8 Further Questions $\quad 40$

\section{Introduction}

In [5], the second-named author defines for each affine Weyl group an affine variety whose integral points are in bijection with the group. The irreducible components of the variety are affine subspaces and are in bijection with the alcoves in a certain polytope; this set of alcoves is partially ordered: the covering relation of this order is determined by applying a reflection sending an alcove to a neighbouring one, further from the origin.

In the case of the affine symmetric group $\widetilde{S}_{n}$, it turns out that the irreducible components are naturally in bijection with the set of circular permutations in $S_{n}$ (that is, $n$-cycles), so that one obtains an order on this set. It is this poset, with its three instances, that we study in the present article.

The article is self-contained and may be read independently of the motivating article [5]. We begin by defining a partial order on the set of circular permutations in $S_{n}$, obtaining a ranked poset (Corollary 4), whose rank function has image the interval $\left\{0,1, \ldots,\left(\begin{array}{l}n \\ 3\end{array}\right)\right\}$. Note the rather unusual maximum rank, which is cubic in $n$. The rank function is computed using inversions in a rather subtle way: it is a signed count of inversions (see Eq.(1)), and it is clearly not the usual length function in the symmetric group).

The edges of the Hasse diagram of this order are indexed by transpositions $(i, j)$ with $i+1<j$; the covering relation conjugates two circular permutations by this transposition, under the condition that the smaller permutation sends $j$ onto $i$ (we call this large circular descent, see Definition 26). The smallest element in the poset is $(1,2, \ldots, n)$ and the largest one is its inverse. Inversion and conjugation by the longest element in $S_{n}$ are anti-automorphisms of the poset. See Figures 1 and 2.

Admitted vectors, introduced by the second-named author in [5], are vectors $v$ of natural integers, indexed by the transpositions considered above, which satisfy the conditions

$$
\left\{\begin{array}{l}
v_{i j}+v_{j k} \leqslant v_{i k} \leqslant v_{i j}+v_{j k}+1, \\
v_{i, i+1}=0 \quad \text { for all } 1 \leqslant i<n .
\end{array} \text { for all } i<j<k,\right.
$$

Admitted vectors are naturally ordered, and we show that the poset of admitted vectors is isomorphic with the previous poset of circular permutations (Theorem 12).

Using the isomorphism of posets, we derive several properties. The poset is a lattice, and we give an algorithmic construction for the supremum and the infimum of two elements, using the vector incarnation of the poset (Theorem 23). The number of edges in the Hasse diagram are counted by Eulerian numbers: precisely, the number of circular 
permutations in $S_{n+1}$ which is covered by $k$ circular permutations is the Eulerian number $a(n, k)$ (= number of permutations in $S_{n}$ having $k$ descents) (Theorem 28); in particular, the number of join-irreducible elements is $2^{n}-n-1$; the poset is however not isomorphic to $S_{n}$ with the weak order: indeed the maximum rank is cubic in $n$, whereas the maximum rank for the weak order is quadratic. We consider also the limit poset when $n \rightarrow \infty$ : that is, we show that for any $k$, and $n$ large enough, the posets coincide in their elements of rank $\leqslant k$; and the limit of the posets is Young's lattice of partitions (Theorem 32).

We give a link with triangulations of an $n$-gon. We consider the functions $\delta_{i j k}$, derived from the above defining identities of admitted vectors, that is,

$$
\delta_{i j k}(v)=v_{i k}-v_{i j}-v_{j k}, i<j<k .
$$

These functions take their values in $\{0,1\}$, and satisfy the Ptolemy-like relation

$$
\delta_{i j k}+\delta_{i k l}=\delta_{i j l}+\delta_{j k l}, i<j<k<l .
$$

We show that, given any triangulation of the $n$-gon, the component indexed by $(1, n)$ of any admitted vector $v$ is equal to the sum of all $\delta_{i j k}(v)$, where the sum is over all triangles $i, j, k$ of the triangulation (Theorem 22).

The third incarnation of the poset, close to the initial motivation [5], is an interval in the affine symmetric group $\tilde{S}_{n}$. We construct a special element $f_{c}$ in this group, and show that our poset is isomorphic with the interval [id, $f_{c}$ ] with the left weak order (Theorem 36). The rank function is therefore the length function in this Coxeter group.

From this isomorphism we derive several consequences: by a result of Reading and Speyer, the lattice is semidistributive (but not distributive, nor modular) (Corollary 44); and by a result of Björner and Brenti, the Möbius function takes values in $\{-1,0,1\}$ (see end of Subsection 6.2).

Finally, in Section 7, we illustrate the bijection between circular permutations and admitted vectors using circular line diagrams.

\section{Ordering circular permutations}

In this section we define the poset which is the topic of the whole article. It is defined first on circular permutations, by way of the covering relation. For each circular permutation, there are as many covers as "large circular descents". The rank function of the poset is computed.

A conjugate of word $w$ is a word $w^{\prime}$ such that for some words $x, y$, one has $w=x y, w^{\prime}=$ $y x$. A factor of a word $w$ is a word $u$ such that $w=x u y$ for some words $x, y$; a circular factor of $w$ is a factor of some conjugate of $w$. We say that the word $s r$ of length 2 is a subword of $w$ if $w=x$ syr $z$ for some words $x, y, z$.

For example, the alphabet being $\{1,2,3, \ldots\}$, the word $u=213$ is a factor of the word $w=21354$, the word 25 is a subword of length 2 of $w$ and 542 is a circular factor of $w$.

For any permutation $w \in S_{n}$, that we view as a word on the alphabet $\{1, \ldots, n\}$, and for any $i, j$ with $1 \leqslant i<j \leqslant n$, define the function $\gamma_{i j}(w)=1$ if $j$ appears before $i$ in $w$, 
and 0 otherwise; that is, $\gamma_{i j}(w)=1$ if $j i$ is an inversion by value in $w$ (which means that $j i$ is a subword of the word $w$ ), and $=0$ if not. Then we define

$$
N(w)=\sum_{1 \leqslant k \leqslant n-1} k(n-k) \gamma_{k, k+1}(w)-\sum_{1 \leqslant i<j \leqslant n} \gamma_{i j}(w) .
$$

For example, $n=5, w=14325$, with inversions $32,43,42$, so that $N(14325)=$ $2 \cdot(5-2)+3 \cdot(5-3)-1-1-1=9$.

Proposition 1. (i) If $w, w^{\prime} \in S_{n}$ are conjugate words, then $N(w)=N\left(w^{\prime}\right)$.

(ii) Suppose that $w$ has the circular factor $s r, s, r \in\{1,2, \ldots, n\}$, with $s>r+1$ and let $w^{\prime \prime}$ be obtained from $w$ by exchanging $r$ and $s$. Then $N\left(w^{\prime \prime}\right)=N(w)+1$.

(iii) $N(12 \cdots n)=0$.

(iv) $N(n \cdots 21)=\left(\begin{array}{l}n \\ 3\end{array}\right)$.

Proof. (i). It suffices to prove this in the case where $w=r u, w^{\prime}=u r$, for $r \in\{1, \ldots, n\}$. By inspection of the inversions of $w$ and $w^{\prime}$, we see that: for any $i<r, w$ has the inversion ri, but $w^{\prime}$ not; for any $j>r, w^{\prime}$ has the inversion $j r$, but $w$ not; all other inversion are identical in $w$ and $w^{\prime}$. Finally we synthesize this for all $1 \leqslant i<r<j \leqslant n$ and $k, l \neq r$ by:

Thus it follows that

$$
\left\{\begin{array}{cc}
\gamma_{r j}(w)=0 & \text { and } \gamma_{i r}(w)=1 \\
\gamma_{r j}\left(w^{\prime}\right)=1 & \text { and } \gamma_{i r}\left(w^{\prime}\right)=0 \\
& \gamma_{k l}(w)=\gamma_{k l}\left(w^{\prime}\right)
\end{array}\right.
$$

$$
\begin{aligned}
N(w)-N\left(w^{\prime}\right)= & (r-1)(n-(r-1)) \gamma_{r-1, r}(w)-\sum_{i=1}^{r-1} \gamma_{i, r}(w)- \\
& r(n-r) \gamma_{r, r+1}\left(w^{\prime}\right)+\sum_{j=r+1}^{n} \gamma_{r j}\left(w^{\prime}\right) \\
& =r(n-r)-(r-1)(n-r+1)-(n-r)+(r-1) \\
& =r n-r^{2}-r n+r^{2}-r+n-r+1-n+r+r-1 \\
& =0 .
\end{aligned}
$$

(ii). By (i), we may assume that $s r$ is a factor of $w$. Since $s>r+1$ it follows that $k(k+1)$ and $(k+1) k$ are simultaneously subwords of $w$ and $w^{\prime \prime}$, or not; moreover, there is one subword of the form $j i, j>i$, less in $w^{\prime \prime}$ than in $w$; hence $N\left(w^{\prime \prime}\right)=N(w)+1$.

(iii). This is straightforward.

(iv). Recall that the sum of the integers from 1 to $n-1$ (resp. of their squares) is equal to $(n-1) n / 2$ (resp. $(n-1) n(2 n-1) / 6)$. Since $\gamma_{i j}(n \ldots 21)=1$ for all $i<j$, it follows that

$$
N(n \ldots 21)=\sum_{k=1}^{n-1} k(n-k)-\sum_{1 \leqslant i<j \leqslant n} 1
$$




$$
\begin{aligned}
& =n \sum_{k=1}^{n-1} k-\sum_{k=1}^{n-1} k^{2}-\frac{n(n-1)}{2} \\
& =\frac{n^{2}(n-1)}{2}-\frac{n(n-1)(2 n-1)}{6}-\frac{n(n-1)}{2} \\
& =\frac{n(n-1)(n-2)}{6} .
\end{aligned}
$$

In this article we call circular permutation an $n$-cycle in $S_{n}$. Such a permutation is denoted in cyclic form by $(w)$, for some $w \in S_{n}$, viewed as a word. For example (12345) denotes the canonical 5-cycle; sometimes we use commas, if necessary, e.g. $(1,2,3,4,5)$.

It is well-known that a circular permutation $(w)$ in $S_{n}$ has exactly $n$ such representations, which are the $\left(w^{\prime}\right), w^{\prime}$ a conjugate of $w$. In particular, there is exactly one representation beginning by 1 , that is $\left(w^{\prime}\right)$ with $w^{\prime}=1 v$.

Corollary 2. The function $N$ induces a function on the set of circular permutations in $S_{n}$.

We denote this function on circular permutations also by $N$. The corollary is illustrated by the following commutative diagram:

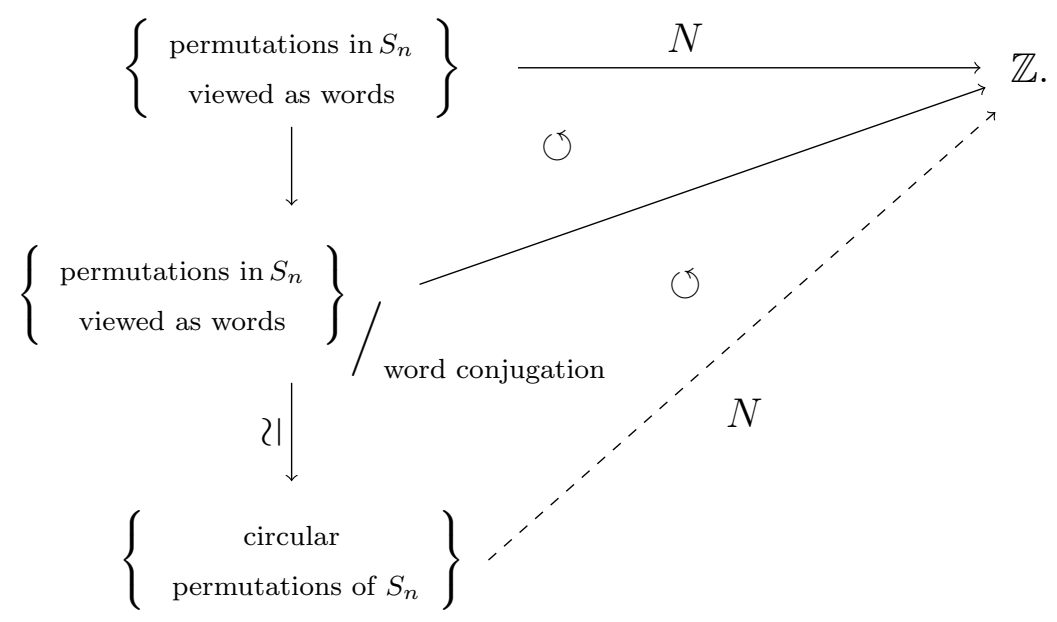

Definition 3 (Relation on circular permutations). Define a relation on circular permutation by $(w) \rightarrow\left(w^{\prime}\right)$ if $w$ has some circular factor $s r$ with $s>r+1$ and $w^{\prime}$ is obtained by replacing in $w$ this factor by $r s$.

It is easy to verify that this relation does not depend on the chosen representatives of the circular permutations. For example, with $s r=51,(12345) \rightarrow(52341)$ is seen also by $(34512) \rightarrow(34152)$.

Corollary 4. The reflexive and transitive closure of the relation $\rightarrow$ is a partial order on the set of circular permutations. The corresponding poset is graded by $N$, its smallest element is $(12 \cdots n)$ and its largest one is $(n \cdots 21)$. 
Proof. There is no cycle in the transitive closure of $\rightarrow$, by Proposition 1 (ii). Thus it is an order.

Note that if for a circular permutation $(w), w$ has the property of having no circular factor $s r$ with $s>r+1$, then $(w)=(n \cdots 21)$ : indeed we may assume that $w$ begins by $n$, and then the second letter must be $n-1$, the third one must be $n-2$, and so on. The element $(n \cdots 21)$ is the maximum of the poset; indeed, it is the only one having the previous property; moreover, any element $\sigma$ having some circular factor $s r$ (with $s>r+1)$ is smaller than some other element, by the definition of $\rightarrow$, so that by iteration, $\sigma<(n \cdots 21)$.

Similarly, $(12 \cdots n)$ is the smallest element of the poset.

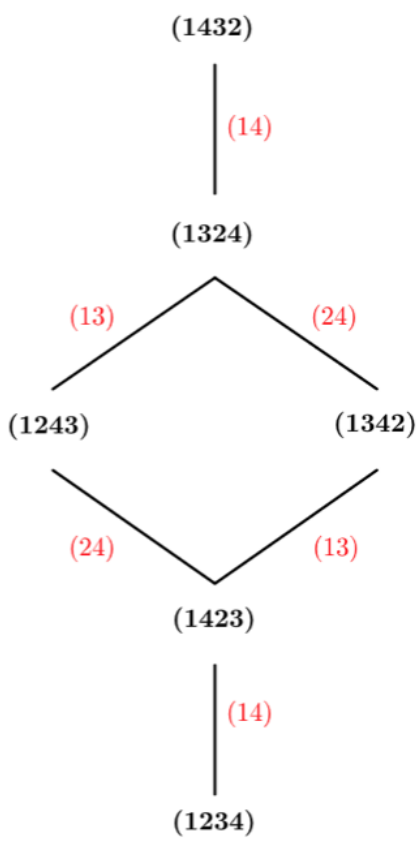

Figure 1: Poset of circular permutations for $n=4$. Red labels indicate the cover relation, which is the conjugation. Ex: $(14)(1234)(14)=(1423)$.

Corollary 5. The image of the function $N$ is $\left\{0,1, \ldots,\left(\begin{array}{l}n \\ 3\end{array}\right)\right\}$.

Proof. This follows from the previous corollary and from Proposition 1.

Remark 6. It turns out that our poset gives a direct answer to a question proposed in the problem 2 on the USAMO 2010 [1]. This problem is the following one: say there are $n$ students standing in a circle, one behind the other. The students have heights $h_{1}<h_{2}<\cdots<h_{n}$. If a student with height $h_{k}$ is standing directly behind a student with height $h_{k-2}$ or less, the two students are permitted to switch places. Prove that it is 


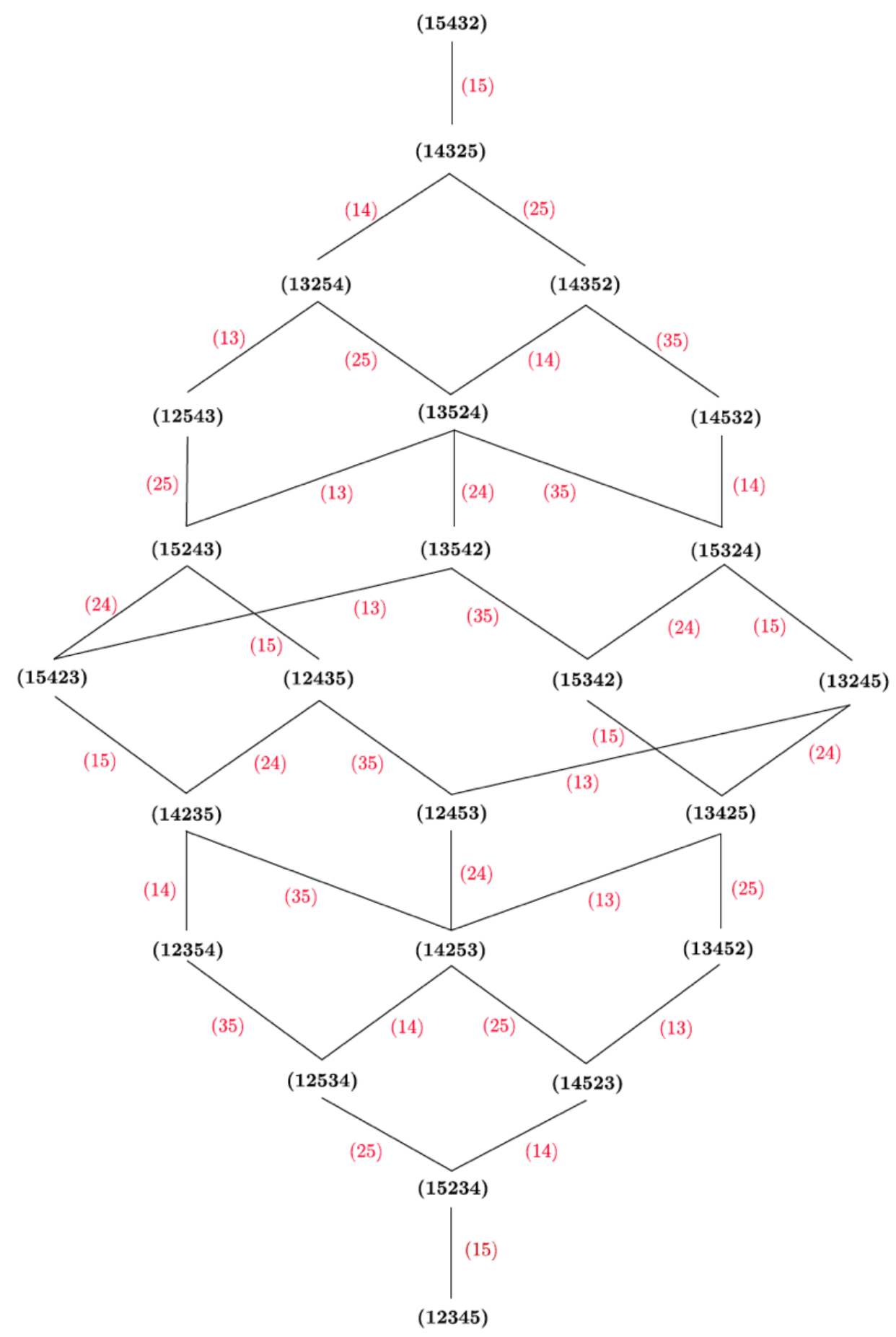

Figure 2: Poset of circular permutations for $n=5$. For the significance of the red labels, see Figure 1. 
not possible to make more than $\left(\begin{array}{l}n \\ 3\end{array}\right) n$ such switches before reaching a position in which no further switches are possible.

Corollary 7. The functions $\sigma \mapsto \sigma^{-1}$ and $\sigma \mapsto w_{0} \circ \sigma \circ w_{0}$ (where $w_{0}=n \cdots 21$ is the longest permutation) are anti-automorphisms of the poset.

In Figures 1 and 2, the first automorphism corresponds to a central symmetry of the Hasse diagram.

Proof. 1. For inversion, this follows from the fact that the inverse of $(w)$ is $(\tilde{w})$, where $\tilde{w}$ is the reversal of $w$. Therefore, cyclic factors $r s$ and $s r$ are interchanged in $(w)$ and its inverse, showing that $(u) \rightarrow(v)$ if and only if $(\tilde{v}) \rightarrow(\tilde{u})$.

2. Note that a cyclic factor $r s$ gives, after applying the second function the cyclic factor $(n+1-r)(n+1-s)$, which implies the result.

Remark 8. In the Hasse diagram of the poset of circular permutations, each edge is labelled by a transposition $(r s), r+1<s$, which is defined by the fact that the largest permutation $\tau$ of this edge has the circular factor $r s$, and the smallest $\sigma$ has the circular factor $s r$ (see Figures 1 and 2); this transposition clearly conjugates both permutations: $\tau=(r s) \circ \sigma \circ(r s)$. Taking any upwards path in this diagram, from a circular permutation $\sigma$ to a larger one, $\tau$ say, we may perform the corresponding product of transpositions in $S_{n}$ from right to left, obtaining a permutation $\alpha$, which conjugates them: $\tau=\alpha \circ \sigma \circ \alpha^{-1}$. This implies that the class of $\alpha$ modulo the centralizer of $\sigma$ (which has $n$ elements) does not depend on the chosen path. But we may be more precise.

Proposition 9. With the previous notations, $\alpha$ depends only on $\sigma$ and $\tau$ and not on the chosen path. In particular, for a maximal path (thus $\sigma=(12 \cdots n)$ and $\tau=(n \cdots 21)=$ $\left.\sigma^{-1}\right)$, one has, as words, $\alpha=n \cdots 21$ if $n$ is odd, and $(n / 2) \cdots 21 n \cdots(1+n / 2)$ if $n$ is even.

As examples, for $n=5$ and 6 the latter permutations are 54321 and 321654 .

The proof of the proposition will be given at the end of Section 3 .

\section{An isomorphism towards admitted vectors}

Admitted vectors are vectors of natural integers, indexed by the set $T$ of transpositions in $S_{n}$, which satisfy certain inequalities. They inherit the partial order of $\mathbb{N}^{T}$. The poset thus obtained is shown to be isomorphic to the poset of circular permutations of the previous section.

Define $S=\{(i, i+1), i=1, \ldots, n-1\}, T_{1}=\{(i, j), 1 \leqslant i, i+1<j \leqslant n\}$ and $T=S \cup T_{1}$. For a vector $v$ in $\mathbb{N}^{T}$, we write $v_{i j}:=v_{(i, j)}$. We say that $v \in \mathbb{N}^{T}$ is admitted if $v_{i, i+1}=0$ for any $i$, and if for any $(i, k) \in T_{1}$, one has

$$
v_{i j}+v_{j k} \leqslant v_{i k} \leqslant v_{i j}+v_{j k}+1 \text { for all } i<j<k .
$$

Note that equivalently $v_{i k}-v_{i j}-v_{j k}=0$ or 1 . 
We show in Figures 3 and 4 all possible admitted vectors for $n=4,5$; we draw there the admitted vectors using a triangle, where the way to place the coordinates is explained in the figures (the coordinates $v_{i, i+1}$ are not drawn, since they are equal to 0 by definition).

The set of admitted vectors inherits the natural partial order of $\mathbb{N}^{T}$. See Figures 3 and 4 , where are drawn the Hasse diagrams of these posets.

We define a mapping $V_{0}$ from $S_{n}$ into $\mathbb{Z}^{T}$ as follows: let $w \in S_{n}$ and let $V_{0}(w)=v \in \mathbb{Z}^{T}$ be defined by

$$
v_{i j}=-\gamma_{i j}(w)+\sum_{i \leqslant k<j} \gamma_{k, k+1}(w)
$$

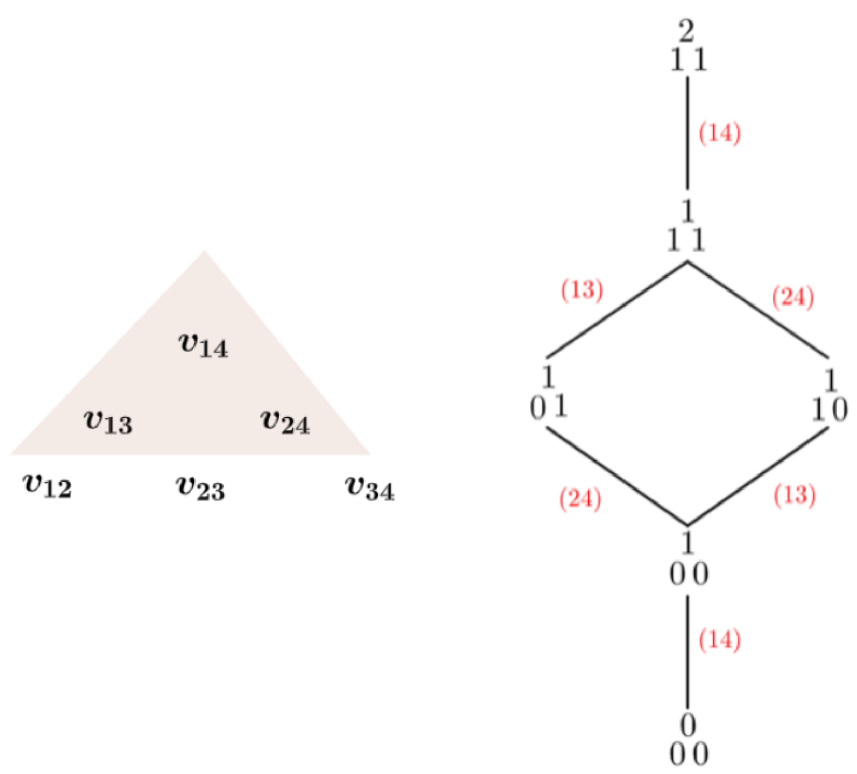

Figure 3: Poset of admitted vectors for $n=4$. The pyramid shows the position of each coefficients but knowing the fact that by definition $v_{i, i+1}=0$ for all $i$ we only keep the ones in the pink pyramid.

Proposition 10. Each $V_{0}(w), w \in S_{n}$, is an admitted vector; $V_{0}(w)=V_{0}\left(w^{\prime}\right)$ if $w, w^{\prime}$ are conjugate; and $N(w)$ is equal to the sum of the components of $V_{0}(w)$.

Proof. Let $v=V_{0}(w)$. Let $1 \leqslant i<j \leqslant n$.

If $(i, i+1) \in S$, then by definition of $V_{0}, v_{i, i+1}=-\gamma_{i, i+1}(w)+\gamma_{i, i+1}(w)=0$. If $(i, k) \in T$, and $i<j<k$, then

$$
v_{i k}-v_{i j}-v_{j k}=-\gamma_{i k}(w)+\sum_{i \leqslant p<k} \gamma_{p, p+1}(w)+\gamma_{i j}(w)-
$$




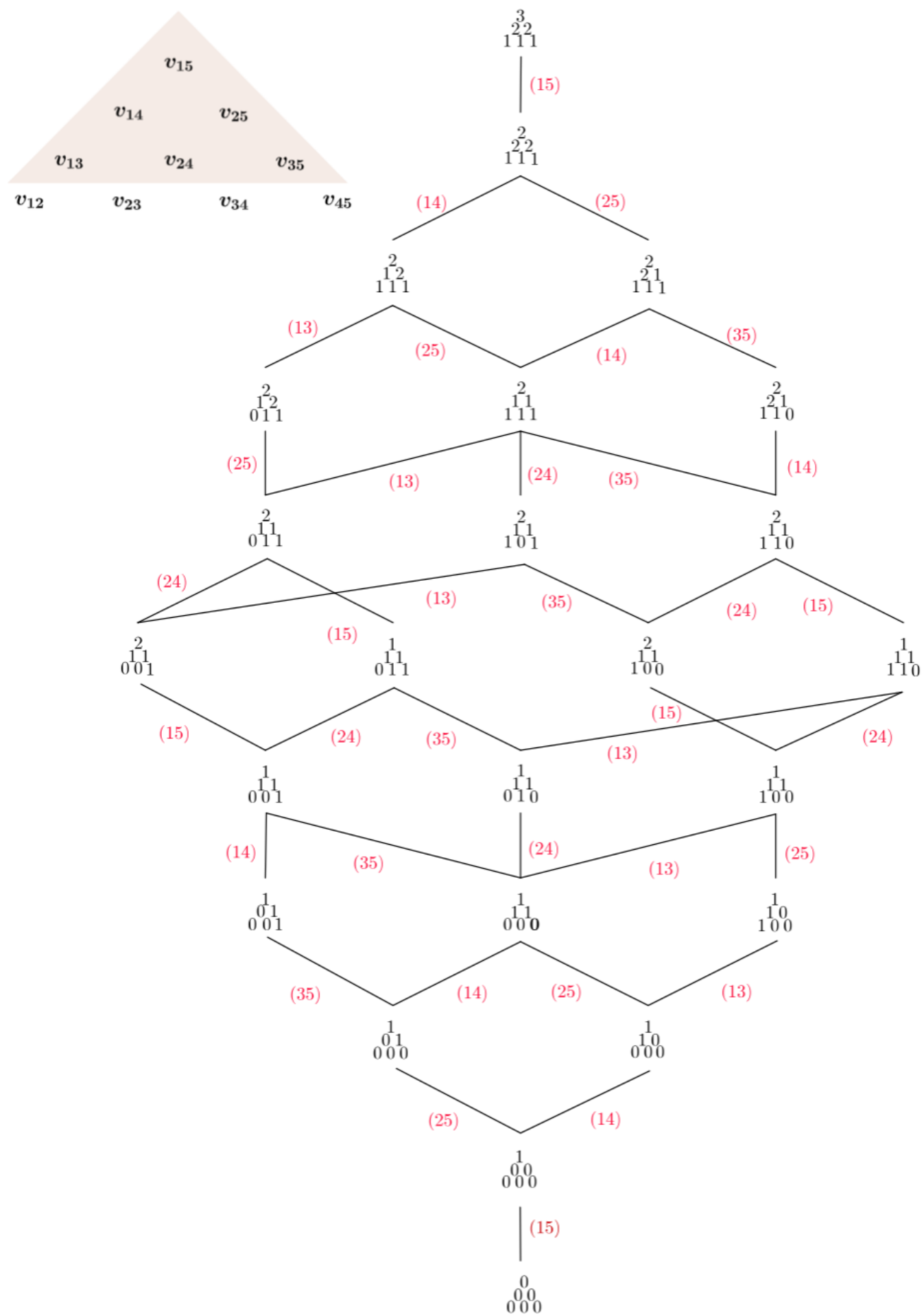

Figure 4: Poset of admitted vectors for $n=5$. The pyramid shows the position of each coefficients but knowing the fact that by definition $v_{i, i+1}=0$ for all $i$ we only keep the ones in the pink pyramid. 


$$
\begin{gathered}
\sum_{i \leqslant p<j} \gamma_{p, p+1}(w)+\gamma_{j k}(w)-\sum_{j \leqslant p<k} \gamma_{p, p+1}(w) \\
=\gamma_{i j}(w)+\gamma_{j k}(w)-\gamma_{i k}(w) .
\end{gathered}
$$

By inspecting the relative positions of $i, j, k$ in the word $w$, one checks that the latter quantity is equal to 0 or 1 . Hence $v$ satisfies the inequalities (2). Moreover, $v_{i, i+1}=0$, and by induction on $j-i$, these inequalities imply that the $v_{i j}$ are all nonnegative.

This proves that $v=V_{0}(w)$ is an admitted vector. We show now that it is invariant under conjugation of $w$. Then

Suppose that $w=r u, w^{\prime}=u r, r \in\{1, \ldots, n\}$. Let $v=V_{0}(w), v^{\prime}=V_{0}\left(w^{\prime}\right)$. Let $i<j$.

$$
v_{i j}=-\gamma_{i j}(w)+\sum_{i \leqslant k<j} \gamma_{k, k+1}(w) \text { and } v_{i j}^{\prime}=-\gamma_{i j}\left(w^{\prime}\right)+\sum_{i \leqslant k<j} \gamma_{k, k+1}\left(w^{\prime}\right) .
$$

In the case $j=i+1$, we certainly have $v_{i j}=v_{i j}^{\prime}$, since they are both equal to 0 .

Thus we may assume that $j>i+1$. Suppose that $i, j \neq r$. If $i<r<j$, there is the subword $r(r-1)$ in $w$, but not in $w^{\prime}$, and there is the subword $(r+1) r$ in $w^{\prime}$, but not in $w$; thus $v_{i j}=v_{i j}^{\prime}$. If on the other hand, the double inequality $i<r<j$ does not hold, then the two previous sums are identically equal and $v_{i j}=v_{i j}^{\prime}$, too.

Suppose that $r=i$. Then $\gamma_{i j}(w)=0, \gamma_{i j}\left(w^{\prime}\right)=1$, and $\gamma_{i, i+1}(w)=0, \gamma_{i, i+1}\left(w^{\prime}\right)=1$; the other terms are identical for $w$ and $w^{\prime}$, therefore $v_{i j}=v_{i j}^{\prime}$. The case $r=j$ is similar.

We deduce that $V_{0}(w)=V_{0}\left(w^{\prime}\right)$. Thus $V_{0}(w)$ is invariant under conjugation of $w$.

Finally, we have

$$
\begin{aligned}
\sum_{i<j} v_{i j} & =\sum_{i<j}\left(-\gamma_{i j}(w)+\sum_{i \leqslant k<j} \gamma_{k, k+1}(w)\right) \\
& =-\sum_{i<j} \gamma_{i j}(w)+\sum_{k=1}^{n} \sum_{i \leqslant k<j} \gamma_{k, k+1}(w) \\
& =-\sum_{i<j} \gamma_{i j}(w)+\sum_{1 \leqslant k \leqslant n} k(n-k) \gamma_{k, k+1}(w) \\
& =N(w) .
\end{aligned}
$$

Definition 11. We define the functions $\delta_{i j k}: \mathbb{N}^{T} \rightarrow \mathbb{N}, 1 \leqslant i \leqslant j \leqslant k \leqslant n$, by $\delta_{i j k}(v)=v_{i k}-v_{i j}-v_{j k}$, where we put $v_{i i}=0$.

These functions satisfy the following relation, for any $i \leqslant j \leqslant k \leqslant l$ :

$$
\delta_{i j k}(v)+\delta_{i k l}(v)=\delta_{i j l}(v)+\delta_{j k l}(v) .
$$

Indeed, the left-hand side evaluated on the admitted vector $v$ is equal to $v_{i k}-v_{i j}-$ $v_{j k}+v_{i l}-v_{i k}-v_{k l}$, whereas the right-hand side gives $v_{i l}-v_{i j}-v_{j l}+v_{j l}-v_{j k}-v_{k l}$, which are both equal to $v_{i l}-v_{i j}-v_{j k}-v_{k l}$. 
Theorem 12. The mapping $V_{0}$ induces a graded poset isomorphism $V$ from the set of circular permutations in $S_{n}$ into the set of admitted vectors in $\mathbb{N}^{T}$, ordered componentwise. The inverse mapping is completely determined as follows: let $w=1 w^{\prime} \in S_{n}$, viewed as word; then $V_{0}(w)=v$ implies that for any $1 \leqslant i<j \leqslant n$, one has $\gamma_{i j}(w)=\delta_{1 i j}(v)$.

The theorem is illustrated for $n=4$ in Figure 5: each red labels represents, from left to right, the coordinate which is increased in the cover relation (with the pyramidal convention of Figure 3); the conjugating transposition; the large circular descent.
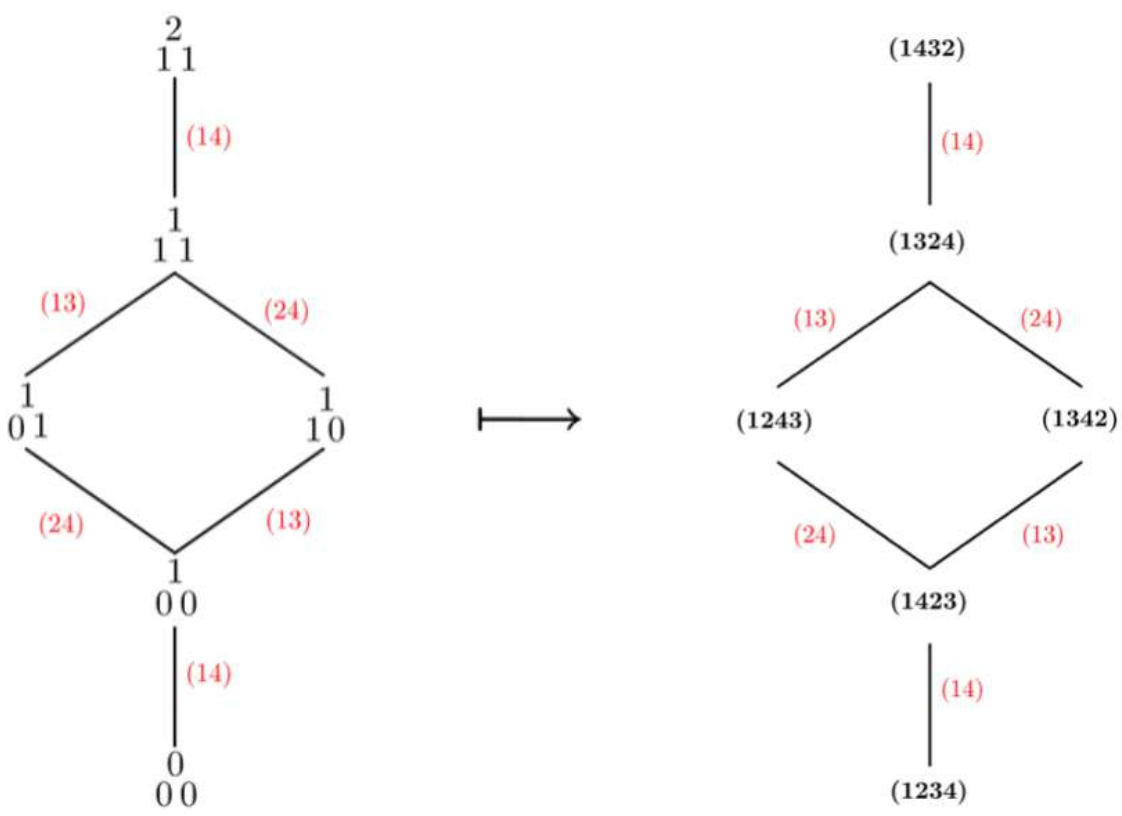

Figure 5: Isomorphism from admitted vectors onto circular permutations in $S_{4}$.

The statement of Theorem 12 uses implicitly the well-known result that a permutation $w \in S_{n}$ is completely determined by its sequence of inversions $\left(\gamma_{i j}(w)\right)_{1 \leqslant i<j \leqslant n}$.

In order to prove Theorem 12 we need several lemmas. Before stating and proving them, we note that the inequalities (2) characterizing admitted vectors are equivalent to the condition that the image of each function $\delta_{i j k}$ is contained in $\{0,1\}$.

Lemma 13. If $u, v$ are admitted vectors such that $u<v$, then for some $1 \leqslant i<j \leqslant n$, one has

$$
\begin{aligned}
& i<p<j \Rightarrow \delta_{i p j}(u)=0 \\
& 1 \leqslant p<i \Rightarrow \delta_{p i j}(u)=1 \\
& j<p \leqslant n \Rightarrow \delta_{i j p}(u)=1 .
\end{aligned}
$$


Proof. In the whole proof we use the fact that the functions $\delta$ map $u$ and $v$ onto 0 or 1 .

1. We show first that there exists a pair $i<j$ such that $\delta_{i p j}(v)=0$ for any $i<p<j$. We construct below a nonempty set $E$, whose minimal elements, for a certain order, are such pairs.

The order on the set of pairs $(i, j), i<j$, is as follows: $(i, j) \leqslant\left(i^{\prime}, j^{\prime}\right)$ if the interval $[i, j]$ is contained in the interval $\left[i^{\prime}, j^{\prime}\right]$ (that is, if $i^{\prime} \leqslant i$ and $j \leqslant j^{\prime}$ ).

Consider the set $E$ of pairs $(i, j), i<j$, such that $u_{i j}<v_{i j}$. Note that by assumption $E$ is nonempty. Let $(i, j)$ be a minimal element in this set, for the previous order. Then for any $\left(i^{\prime}, j^{\prime}\right)<(i, j)$, one has by minimality $u_{i^{\prime}, j^{\prime}}=v_{i^{\prime}, j^{\prime}}$; in particular, for $i<p<j$, $u_{i p}=v_{i p}$ and $u_{p j}=v_{p j}$. Moreover by construction, $u_{i j}-v_{i j} \leqslant-1$. Since $\delta_{i p j}(v) \leqslant 1$, it follows that:

$$
\begin{aligned}
\delta_{i p j}(u) & =u_{i j}-u_{i p}-u_{p j} \\
& =u_{i j}-v_{i j}+v_{i j}-v_{i p}-v_{p j} \\
& =\left(u_{i j}-v_{i j}\right)+\delta_{i p j}(v) \\
& \leqslant 0 .
\end{aligned}
$$

Thus $\delta_{i p j}(u)=0$.

2. We consider now that set $F$ of pairs $(i, j), i<j$, such that for any $i<p<j$, one has $\delta_{i p j}(u)=0$. This set is nonempty by 1 . Let $(i, j)$ be a maximal element in this set. We claim that for any $1 \leqslant p<i$, one has $\delta_{p i j}(u)=1$ and for any $n \geqslant p>j, \delta_{i j p}(u)=1$. The claim implies the lemma.

Suppose by contradiction that the claim is not true; then by symmetry of the two cases, we may assume that for some $n \geqslant l>j$, one has $\delta_{i j l}(u)=0$, and we choose $l$ minimum.

Let $j<k<l$. By minimality of $l$, we have $\delta_{i j k}(u)=1$. Then by Eq. $(3), \delta_{i j k}(u)+$ $\delta_{i k l}(u)=\delta_{i j l}(u)+\delta_{j k l}(u)$. Since $\delta_{i j k}(u)=1$ and $\delta_{i j l}(u)=0$, we must have $\delta_{i k l}(u)=0$.

Now let $i<p<j$. Therefore, by Eq.(3) applied to $i<p<j<l$, we have $\delta_{i p j}(u)+$ $\delta_{i j l}(u)=\delta_{i p l}(u)+\delta_{p j l}(u)$. Since $\delta_{i j l}(u)=0$ and $\delta_{i p j}(u)=0$, we must have $\delta_{i p l}(u)=0$.

We conclude that for any $p$ with $i<p<l$, we have $\delta_{i p l}(u)=0$. Since $(i, j)<(i, l)$, this contradicts the maximality of the pair $(i, j)$.

Lemma 14. Let $v$ be an admitted vector, let $i<j$ and define $v^{\prime}$ by $v_{i j}+1=v_{i j}^{\prime}$, whereas $v_{r s}=v_{r s}^{\prime}$ for the other coordinates. Then the two following conditions are equivalent:

(i) $v^{\prime}$ is an admitted vector;

(ii) the following equations hold:

$$
\begin{aligned}
& i<p<j \Rightarrow \delta_{i p j}(v)=0 \\
& 1 \leqslant p<i \Rightarrow \delta_{p i j}(v)=1 \\
& j<p \leqslant n \Rightarrow \delta_{i j p}(v)=1 .
\end{aligned}
$$


Proof. Note that

$$
\begin{gathered}
i<p<j \Rightarrow \delta_{i p j}(v)=v_{i j}-v_{i p}-v_{p j}=v_{i j}^{\prime}-1-v_{i p}^{\prime}-v_{p j}^{\prime}=\delta_{i p j}\left(v^{\prime}\right)-1 ; \\
1 \leqslant p<i \Rightarrow \delta_{p i j}(v)=v_{p j}-v_{p i}-v_{i j}=v_{p j}^{\prime}-v_{p i}^{\prime}-v_{i j}^{\prime}+1=\delta_{p i j}\left(v^{\prime}\right)+1 \\
j<p \leqslant n \Rightarrow \delta_{i j p}(v)=\delta_{i j p}\left(v^{\prime}\right)+1
\end{gathered}
$$

In all other cases, $\delta_{r s t}(v)=\delta_{r s t}\left(v^{\prime}\right)$.

(i) implies (ii): let $i<p<j$; then by the above calculations $\delta_{i p j}(v)=\delta_{i p j}\left(v^{\prime}\right)-1$; since the values of $\delta$ are 0 or 1 , we must have $\delta_{i p j}(v)=0$. Let $p<i$; then $\delta_{p i j}(v)=\delta_{p i j}\left(v^{\prime}\right)+1$; this forces $\delta_{p i j}(v)=1$. Similarly, $p>j$ implies $\delta_{i j p}(v)=1$. Hence equations (4) hold.

(ii) implies (i): It is enough to prove that the functions $\delta$ take the values 0 or 1 when evaluated on $v^{\prime}$. Let $i<p<j$; then $\delta_{i p j}(v)=\delta_{i p j}\left(v^{\prime}\right)-1$, hence $\delta_{i p j}\left(v^{\prime}\right)=1$ by Eq.(4). Let $1 \leqslant p<i$; then $\delta_{p i j}(v)=\delta_{\text {pij }}\left(v^{\prime}\right)+1$, hence $\delta_{\text {pij }}\left(v^{\prime}\right)=0$. Similarly, for $n \geqslant p>j$, $\delta_{i j p}\left(v^{\prime}\right)=0$. In all other cases, $\delta_{r s t}\left(v^{\prime}\right)=\delta_{r s t}(v)=0$ or 1 . This concludes the proof.

Corollary 15. Let $u<v$ be admitted vectors. Then there exists an admitted vector $u^{\prime}$ such that $u<u^{\prime} \leqslant v$ and that for some $1 \leqslant i<j \leqslant n$ one has $u_{i j}+1=u_{i j}^{\prime}$, whereas $u_{r s}=u_{r s}^{\prime}$ for the other coordinates.

Note that this implies that necessarily $u^{\prime}$ covers $u$ for the order of admitted vectors. The corollary could be given a geometric proof, in the spirit of [5], but it follows also directly from the two previous lemmas.

Lemma 16. Let $w \in S_{n}$, viewed as a word. If $1 \leqslant i<j \leqslant n$, then $j i$ is a factor of $w$ if and only if the following conditions hold:

(i) $\gamma_{i j}(w)=1$;

(ii) for any $i<p<j$, $\gamma_{i p}(w)+\gamma_{p j}(w)=1$;

(iii) for any $1 \leqslant p<i, \gamma_{p i}(w)=\gamma_{p j}(w)$;

(iv) for any $n \geqslant p>j, \gamma_{i p}(w)=\gamma_{j p}(w)$.

Proof. Condition (i) means that $j i$ is a subword of $w$. If $1 \leqslant p<i$, then $\gamma_{p i}(w)=\gamma_{p j}(w)=$ 0 means that $p$ is at the left of both $i$ and $j$ in $w$, and $\gamma_{p i}(w)=\gamma_{p j}(w)=1$ means that $p$ is at their right; hence condition (iii) means that $p$ is not between $i$ and $j$ in $w$. Condition (iv) is similar in the case $n \geqslant p>j$. Condition (ii) means similarly that for $i<p<j, p$ is not between $i$ and $j$.

Thus the four conditions together mean that $j i$ is a factor of $w$.

To complete the picture, we give the following dual result, whose proof may be deduced from the proof of Lemma 14:

Lemma 17. Let $v^{\prime}$ be an admitted vector, let $i<j$ and define $v$ by $v_{i j}=v_{i j}^{\prime}-1$, whereas $v_{r s}=v_{r s}^{\prime}$ for the other coordinates. Then the two following conditions are equivalent:

(i) $v$ is an admitted vector; 
(ii) the following equations hold:

$$
\begin{aligned}
& i<p<j \Rightarrow \delta_{i p j}\left(v^{\prime}\right)=1 \\
& 1 \leqslant p<i \Rightarrow \delta_{p i j}\left(v^{\prime}\right)=0 \\
& j<p \leqslant n \Rightarrow \delta_{i j p}\left(v^{\prime}\right)=0 .
\end{aligned}
$$

Proof of Theorem 12. 1. By Proposition 10, $V$ is well-defined on the set of circular permutations.

2. We show that, for $w=1 w^{\prime}$, and $V_{0}(w)=v$, for any $1 \leqslant i<j \leqslant n$, one has $\gamma_{i j}(w)=-v_{i j}+v_{1 j}-v_{1 i}=\delta_{1 i j}(v)$.

In the case $i \neq 1$, we have indeed

$$
\begin{aligned}
\delta_{1 i j}(v)=-v_{i j}+v_{1 j}-v_{1 i} & \\
=\gamma_{i j}(w)-\sum_{i \leqslant k<j} \gamma_{k, k+1}(w)-\gamma_{1 j}(w) & +\sum_{1 \leqslant k<j} \gamma_{k, k+1}(w)+\gamma_{1 i}(w) \\
& -\sum_{1 \leqslant k<i} \gamma_{k, k+1}(w) .
\end{aligned}
$$

The three summations cancel, and $\gamma_{1 j}(w)=0=\gamma_{1 i}(w)$, since $w=1 w^{\prime}$. Thus the previous sum is equal to $\gamma_{i j}(w)$. In case $i=1$, one has

$$
\delta_{1 i j}(v)=-v_{1 j}+v_{1 j}-v_{11}=0=\gamma_{1 j}(w) .
$$

3. This implies that the mapping $V$ is injective, and proves the formulas of the statement.

4. In order to prove surjectivity, let $v$ be an admitted vector. Note that by the inequalities (2), for $i<j$ the numbers $-v_{i j}+v_{1 j}-v_{1 i}=\delta_{1 i j}(v)$ are equal to 0 or 1 . Consider the set $I$ of pairs $(i, j)$ such that $1<i<j \leqslant n$ and $\delta_{1 i j}(v)=1$. We show that this set is the set of inversions by value of some permutation $w$ (meaning that $I$ is the set of $(i, j), i<j$, such that $j i$ is a subword of $w)$.

Recall that a set $I$ of pairs $(i, j), i<j$, is the set of inversions by value of a permutation if and only one has the two conditions: (i) $(i, j)$ and $(j, k) \in I$ implies $(i, k) \in I$; and (ii) if $i<j<k$ and $(i, k) \in I$, then $(i, j)$ or $(j, k)$ is in $I$.

Therefore with $I$ as above, it is enough to prove (i) and (ii).

By Eq.(3), we have $\delta_{1 i j}(v)+\delta_{1 j k}(v)=\delta_{1 i k}(v)+\delta_{i j k}(v)$, and $\delta_{i j k}(v)=0$ or 1 . Therefore, (i) and (ii) easily follow.

5. For the permutation $w$ constructed in 4 , its set of inversions by value is $I=$ $\left\{(i, j), 1<i<j \leqslant n, \delta_{1 i j}(v)=1\right\}$. Therefore $\gamma_{i j}(w)=-v_{i j}+v_{1 j}-v_{1 i}$ for any $1 \leqslant i<j \leqslant 1$ (since it is also true for $i=1$, because by definition of $I$, there is no inversion $1 j$ ). We show that $V(w)=v$. We have indeed

$$
-\gamma_{i j}(w)+\sum_{i \leqslant k<j} \gamma_{k, k+1}(w)=v_{i j}-v_{1 j}+v_{1 i}+\sum_{i \leqslant k<j}\left(-v_{k, k+1}+v_{1, k+1}-v_{1, k}\right)=v_{i j},
$$

since $v_{k, k+1}=0$ and since the summation is a telescoping sum. 
6. We have thus proved that $V$ is a bijection from the set of circular permutations onto the set of admitted vectors. We show now that $V$ is increasing. It is enough to show that if the permutation-word $w$ has the circular factor $j i$ with $j>i+1$ and if $w^{\prime}$ is obtained from $w$ by exchanging $i$ and $j$, then $v^{\prime}=V_{0}\left(w^{\prime}\right)$ is obtained by increasing by 1 the component ij of $v=V_{0}(w)$. Since $V_{0}$ is invariant under conjugation of $w$, we may assume that $j i$ is a factor of $w$.

We have

$$
v_{i j}=-\gamma_{i j}(w)+\sum_{i \leqslant k<j} \gamma_{k, k+1}(w)
$$

and

$$
v_{i j}^{\prime}=-\gamma_{i j}\left(w^{\prime}\right)+\sum_{i \leqslant k<j} \gamma_{k, k+1}\left(w^{\prime}\right) .
$$

Thus $v_{i j}+1=v_{i j}^{\prime}$, since the values of $\gamma_{k, k+1}$ are the same for $w$ and $w^{\prime}$ and since the subword $j i$ disappears from $w$ to $w^{\prime}$. Let now $l>k+1$ and $(k, l) \neq(i, j)$. Similar arguments then show that $v_{k l}=v_{k l}^{\prime}$.

7. To conclude, we must show that if $v=V_{0}(w), v^{\prime}=V_{0}\left(w^{\prime}\right)$ and $v<v^{\prime}$, then $(w)<\left(w^{\prime}\right)$ for the order of circular permutations. In view of Corollary 15, we are reduced to the case where $v$ and $v^{\prime}$ differ by 1 on some coordinate $i j: v_{i j}^{\prime}=v_{i j}+1$ and $v_{k l}=v_{k l}^{\prime}$ if $(k, l) \neq(i, j)$.

We may assume that $w=1 u$, and we show that $j i$ is a circular factor of $w$, which will suffice, by definition of the order on circular permutations and by 6 .

Suppose that $i>1$. We apply Lemma 16 to show that $j i$ is a factor of $w$. We apply below several times Eq.(3), Lemma 14 and 2.

(i) We have $\gamma_{i j}(w)=\delta_{1 i j}(v)=1$.

(ii) Let $i<p<j$. Then we have

$$
\gamma_{i p}(w)+\gamma_{p j}(w)=\delta_{1 i p}(v)+\delta_{1 p j}(v)=\delta_{1 i j}(v)+\delta_{i p j}(v)=1 .
$$

(iii) Let $p<i$. Then

$$
\gamma_{p i}(w)-\gamma_{p j}(w)=\delta_{1 p i}(v)-\delta_{1 p j}(v)=-\delta_{1 i j}(v)+\delta_{p i j}(v)=0 .
$$

(iv) is similar. We conclude that $j i$ is a factor of $w$.

Suppose now that $i=1$. Then we have to show that $j 1$ is a circular factor of $w=1 u$, which means that $j$ is the last letter of $w$. Thus we must prove that for $1<p<j, j p$ is not a subword of $w$, that is $\gamma_{p j}(w)=0$; and if $p>j$, that $j p$ is a not a subword, that is, $p j$ is one, equivalently $\gamma_{j p}(w)=1$. But we have, using Lemma 14: if $p<j$, $\gamma_{p j}(w)=\delta_{1 p j}(v)=0$; and if $p>j, \gamma_{j p}(w)=\delta_{1 j p}(v)=1$, which concludes the proof.

The proof implies the following result, which is illustrated by the labels of the edges in the Hasse diagram of Figures 3 and 4. 
Corollary 18. Let $\sigma, \sigma^{\prime}$ be circular permutations and $v, v^{\prime}$ be the corresponding admitted vectors. Then $\sigma^{\prime}$ covers $\sigma$ if and only if $v^{\prime}$ covers $v$. Moreover the following conditions are equivalent, for $1 \leqslant r, r+1<s \leqslant n$ :

(i) $\sigma^{\prime}$ is obtained from $\sigma$ by replacing the circular factor sr by $r s$;

(ii) $v_{r s}^{\prime}=v_{r s}+1$.

In the next result, we transfer to the poset of admitted vectors the anti-automorphisms of the poset of circular permutations, as they appear in Corollary 7.

\section{Corollary 19.}

1. The anti-automorphism of the poset of admitted vectors, corresponding to inversion in the poset of circular permutations, is defined by $u \mapsto v$, with $v_{i j}=j-i-1-u_{i j}$.

2. The anti-automorphism of the set of admitted vectors, corresponding to conjugation by the longest permutation $w_{0}$, is defined by $u \mapsto v$, with $v_{i j}=j-i-1-u_{n+1-j, n+1-i}$.

Note that the product of the two previous anti-automorphisms of the poset is the automorphism $u \rightarrow v$ with $v_{i j}=u_{n+1-j, n+1-i}$.

Proof. 1. Inversion of circular permutations is the mapping $(w) \mapsto(\tilde{w})$; the corresponding admitted vectors are respectively $u$ and $v$ with

$$
u_{i j}=-\gamma_{i j}(w)+\sum_{i \leqslant k<j} \gamma_{k, k+1}(w)
$$

and

$$
v_{i j}=-\gamma_{i j}(\tilde{w})+\sum_{i \leqslant k<j} \gamma_{k, k+1}(\tilde{w})
$$

Since $\gamma_{r s}(\tilde{w})=1-\gamma_{r s}(w)$, we have

$$
v_{i j}=-\left(1-\gamma_{i j}(w)\right)+\sum_{i \leqslant k<j}\left(1-\gamma_{k, k+1}(w)\right)=-u_{i j}-1+j-i .
$$

2. This anti-automorphism is defined, for circular permutations, by $(w) \mapsto\left(w^{\prime}\right)$, where $w^{\prime}$ is obtained by replacing in $w$ each letter by its complement $n+1-k$. Hence $\gamma_{i j}\left(w^{\prime}\right)=1-\gamma_{n+1-j, n+1-i}(w)$. Therefore, denoting by $u, v$ respectively the admitted vectors corresponding to $(w)$ and $\left(w^{\prime}\right)$, we have

$$
\begin{aligned}
v_{i j} & =-\gamma_{i j}\left(w^{\prime}\right)+\sum_{i \leqslant k<j} \gamma_{k, k+1}\left(w^{\prime}\right) \\
& =-\left(1-\gamma_{n+1-j, n+1-i}(w)\right)+\sum_{i \leqslant k<j}\left(1-\gamma_{n+1-k-1, n+1-k}(w)\right) \\
& =-1+j-i-\left(-\gamma_{n+1-j, n+1-i}(w)+\sum_{l=n+1-j}^{n+1-i} \gamma_{l, l+1}(w)\right) \\
& =j-i-1-u_{n+1-j, n+1-i} .
\end{aligned}
$$


Corollary 20. The smallest admitted vector is the null vector, and the largest one, $w$ say, satisfies $w_{i j}=j-i-1$; moreover, $\delta_{i j k}(w)=1$ for any $i<j<k$.

Proof. Clearly, the null vector is admitted and is the smallest element in the poset of admitted vectors. Using Corollary 19, we see that $w$ as defined in the statement is the largest element. Now

$$
\begin{aligned}
\delta_{i j k}(w) & =w_{i k}-w_{i j}-w_{j k} \\
& =k-i-1-(j-i-1)-(k-j-1) \\
& =1 .
\end{aligned}
$$

Proof of Proposition 9. 1. It is enough to prove this result when $\sigma=(12 \cdots n)$. Consider a path from $\sigma$ to $\tau$, whose successive edges are labelled $\left(r_{1}, s_{1}\right), \ldots,\left(r_{p}, s_{p}\right)$, with $r_{i}+1<s_{i}$, and let $\sigma=\left(w_{0}\right),\left(w_{1}\right), \ldots,\left(w_{p-1}\right),\left(w_{p}\right)=\tau$ the successive vertices in the path, written as $n$-cycles, so that each $w_{i}$ is a word. Then $s_{i} r_{i}$ is a circular factor of the word $w_{i-1}$, and $w_{i}$ is obtained from the latter word by exchanging $r_{i}$ and $s_{i}$ in it.

Define the permutations $\alpha_{i}=\left(r_{i}, s_{i}\right) \circ \cdots \circ\left(r_{1}, s_{1}\right)$. Then $\left(w_{i}\right)=\alpha_{i} \circ \sigma \circ \alpha_{i}^{-1}$. It follows that, in cycle notation, $\left(w_{i}\right)=\left(\alpha_{i}(1) \cdots \alpha_{i}(n)\right)$ and therefore $s_{i+1} r_{i+1}$, being a circular factor of $\left(w_{i}\right)$, is a circular factor of the word $\alpha_{i}$. We have $\alpha_{i+1}=\left(r_{i+1}, s_{i+1}\right) \circ \alpha_{i}$ and thus, as words, $\alpha_{i+1}$ is obtained from $\alpha_{i}$ by exchanging $r_{i+1}$ and $s_{i+1}$, whose positions moreover must differ by 1 in both words (modulo $n$ ). Hence:

$$
\left\{\begin{array}{c}
\alpha_{i+1}^{-1}\left(r_{i+1}\right) \equiv \alpha_{i}^{-1}\left(r_{i+1}\right)-1 \quad \bmod n \\
\alpha_{i+1}^{-1}\left(s_{i+1}\right) \equiv \alpha_{i}^{-1}\left(s_{i+1}\right)+1 \quad \bmod n \\
\alpha_{i+1}^{-1}(j)=\alpha_{i}^{-1}(j) \text { if } j \neq r_{i+1}, s_{i+1} .
\end{array}\right.
$$

It follows by induction that for any $j=1, \ldots, n$, the position of $j$ in the word $\alpha_{i+1}$ is congruent modulo $n$ to: $j$ plus the number of $(r s)$ on the path with $s=j$ minus the number of $(r s)$ on the path with $r=j$. From Corollary 18, it follows that it is congruent modulo $n$ to:

$$
j+\sum_{r<s=j} v_{r s}-\sum_{j=r<s} v_{r s}
$$

where $v$ is the admitted vector associated to the circular permutation $\left(w_{i+1}\right)$.

Therefore each $\alpha_{i+1}$ depends only on $v$, which depends only on $\left(w_{i+1}\right)$; thus $\alpha=\alpha_{p}$ depends only on $\tau$.

2. It remains to determine $\alpha$ for a maximal path in the poset. The previous calculations and Corollary 20 show that $\alpha^{-1}(j)$ is congruent modulo $n$ to $j+\sum_{r<j}(j-r-1)-\sum_{j<s}(s-j-1)$. Moreover, a direct computation shows that:

$$
\begin{aligned}
& j+\sum_{r<j}(j-r-1)-\sum_{j<s}(s-j-1) \\
= & j+((j-2)+\cdots+1+0)-(0+1+\cdots+(n-j-1)) \\
= & j+\frac{(j-2)(j-1)}{2}-\frac{(n-j-1)(n-j)}{2} .
\end{aligned}
$$


The cases $j=1$ and $j=n$ have to be treated separately, but the final formula is correct in these cases as well.

Suppose that $n=2 k+1$. Then using the fact that the inverse of $2 \bmod n$ is $-k$, we find that $\alpha^{-1}(j)$ is congruent modulo $n$ to $j-k(j-2)(j-1)+k j(j+1)$. Hence, omitting the calculations, to $n+1-j$. Thus, as words, $\alpha^{-1}=n \cdots 21$, and $\alpha=\alpha^{-1}$, since it is an involution.

Suppose now that $n=2 k$. Then a calculation left to the reader shows that modulo $n$, $\alpha^{-1}(j)$ is congruent to $k+1-j$. Hence, as word, $\alpha^{-1}=(n / 2) \cdots 21 n \cdots(1+n / 2)$ and $\alpha=\alpha^{-1}$ since it is an involution.

\section{The functions $\delta$ and triangulations of an $n$-gon}

The $n$-gon is a famous object whose combinatorial and geometrical structure appears in a wide variety of subjects. A striking illustration of the ubiquity of the $n$-gon lies for example in the theory of cluster algebras introduced by S. Fomin and A. Zelevinsky in [8]. In this section we relate the $n$-gon with the functions $\delta_{i j k}$ introduced in Definition 11.

We first characterize the sequences $\left(\gamma_{i j}(v)\right)_{1 \leqslant i<j \leqslant n}, v$ an admitted vector, and show that $v$ is completely determined by this sequence. The computation of $v$ will lead to triangulations of an $n$-gon.

Theorem 21. The mapping $v \mapsto\left(\delta_{i j k}(v)\right)_{1 \leqslant i<j<k \leqslant n}$ is a bijection from the set of admitted vectors onto the set of sequences $\left(a_{i j k}\right)_{1 \leqslant i<j<k \leqslant n}$ with values in $\{0,1\}$, satisfying the relation $a_{i j k}+a_{i k l}=a_{i j l}+a_{j k l}$ for any $i<j<k<l$.

Proof. The mapping is well-defined by Eq. (3). Injectivity follows by induction on $j-i$ from the initial conditions $v_{i, i+1}=0$ and if $j-i>1$, from the equation $v_{i j}=v_{i p}+v_{p j}+$ $\delta_{i p j}(v)$ for any $p, i<p<j$.

To prove surjectivity, let $\left(a_{i j k}\right)_{1 \leqslant i<j<k \leqslant n}$ be a sequence, with values in $\{0,1\}$, satisfying the relations $a_{i j k}+a_{i k l}=a_{i j l}+a_{j k l}$ for any $i<j<k<l$. Define $v_{i j}$ by induction: $v_{i, i+1}=0$ and for $j-i>1$, choose $p$ between $i$ and $j$, and define $v_{i j}$ by $v_{i j}=v_{i p}+v_{p j}+a_{i p j}$. We verify, by induction on $j-i$, that this does not depend on the choice of $p$. So let $i<p<q<j$. We have by induction $v_{i q}=v_{i p}+v_{p q}+a_{i p q}$ and $v_{p j}=v_{p q}+v_{q j}+a_{p q j}$. The above relations imply that $a_{i p q}+a_{i q j}=a_{i p j}+a_{p q j}$. Hence

$$
\begin{aligned}
v_{i p}+v_{p j}+a_{i p j} & =v_{i p}+v_{p q}+v_{q j}+a_{p q j}+a_{i p j} \\
& =v_{i p}+v_{p q}+v_{q j}+a_{i p q}+a_{i q j} \\
& =v_{i q}+v_{q j}+a_{i q j}
\end{aligned}
$$

and so $v_{i j}$ is well-defined. The fact that $v$ is an admitted vector then follows from the fact that for any $i<j<k, v_{i k}-v_{i j}-v_{j k}=a_{i j k} \in\{0,1\}$, implying the inequalities (2).

We consider a regular $n$-gon in the plane whose vertices are sequentially numbered $1,2, \ldots, n$. In this $n$-gon, a triangle (resp. convex quadrangle) is determined by a subset of three (resp. four) vertices of the $n$-gon. A triangulation of the $n$-gon is a set of triangles whose interiors do not pairwise intersect, and who cover the whole $n$-gon. 
Note that a 3 -subset $H$ of $\{1,2, \ldots, n\}$ determines uniquely three numbers $i<j<k$ such that $H=\{i, j, k\}$. We write $\delta_{H}:=\delta_{i j k}$.

Theorem 22. If $v$ is an admitted vector, and $\mathcal{T}$ a triangulation of the $n$-gon, then $v_{1 n}$ is equal to the sum of all $\delta_{T}(v)$ for all triangles $T \in \mathcal{T}$.

Proof. Consider the unique triangle $\{1,2, p\}$ in $\mathcal{T}$ (it is the triangle containing the edge 12 of the $n$-gon). By induction, we have $v_{1 p}=\sum_{T} \delta_{T}(v)$, sum over all triangles in $\mathcal{T}$ contained in the sub-p-gon with vertices $1,2, \ldots, p$, which form a triangulation of this $p$ gon. Similarly, the triangles contained in the $n-p+1$-gon with vertices $p, p+1, \ldots, n$ form a triangulation of it; hence by induction (after appropriate reindexing), $v_{p n}=\sum_{T} \delta_{T}(v)$, sum over all triangles of this triangulation. If we add the the triangle with vertices $1, p, n$ to these two triangulations we obtain the original triangulation. Now $v_{1 n}=v_{1 p}+v_{p n}+\delta_{1 p n}$, which concludes the proof.

\begin{tabular}{|c|c|}
\hline$v_{15}$ & 2 \\
\hline$v_{14} v_{25}$ & 11 \\
\hline$v_{13} v_{24} v_{35}$ & $\begin{array}{lll}1 & 0 & 1\end{array}$ \\
\hline${ }_{12} v_{23} v_{34} v_{45}$ & $\begin{array}{lll}0 & 0 & 0\end{array}$ \\
\hline
\end{tabular}

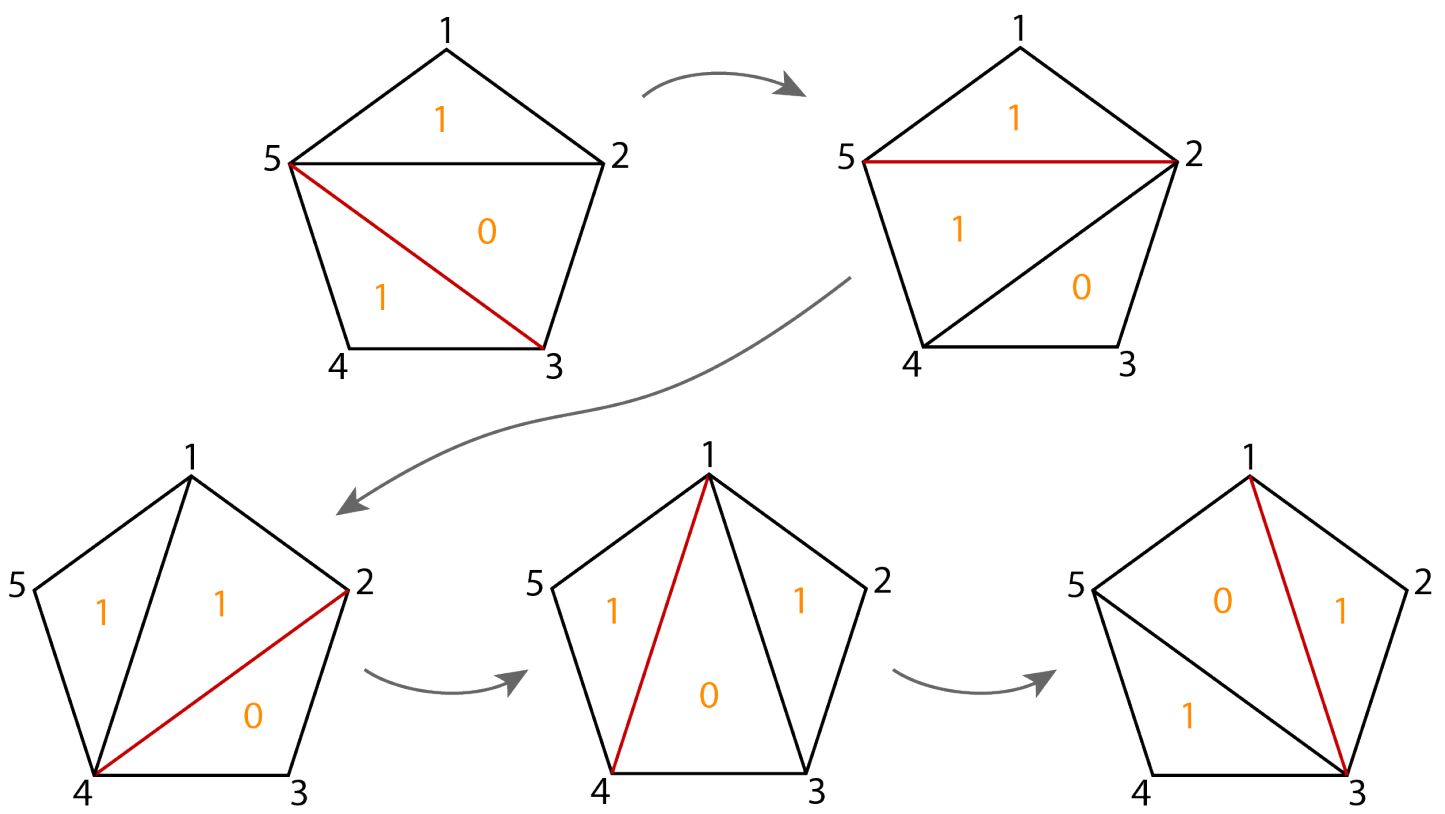

Figure 6: Illustration of Theorem 22 and the discussion following the proof.

More generally, for any $i<j$, the coefficient $v_{i j}$ of an admitted vector $v$ is equal to $\sum_{T} \delta_{T}(v)$, where the sum is over all triangles of a triangulation of the $j-i+1$-gon with vertices $i, i+1, \ldots, j$.

Given a triangulation, consider a convex quadrangle such that one of its diagonal determines two triangles of the triangulation. If we replace in the triangulation these two 
triangles by the two others of the quadrangle, determined by the other diagonal, then we obtain a new triangulation: we say that the new triangulation is obtained by mutation. It is known that given two triangulations of the $n$-gon, one may transform one into the other by a sequence of mutations.

Note that Eq.(3) implies the fact that the sum $\sum_{T} \delta_{T}(v)$, over all triangles in a triangulation, is invariant under mutation.

Theorem 22 is illustrated in Figure 6 . We choose $v$ defined in the figure, and therefore $\delta_{345}=v_{35}-v_{34}-v_{45}=1-0-0=1$; we thus put 1 inside the triangle with vertices $3,4,5$. Similarly for the other triangles. The mutations, with respect to the red triangle sides, are indicated by arrows in the figure. One verifies that for each triangulation, the sum of the labels in the triangles is 2 , thereby confirming the theorem.

We note finally that the sequence $\left(\delta_{i j k}(v)\right)_{1 \leqslant i<j<k \leqslant n}$ is completely defined by the subsequence $\left(\delta_{1 i j}(v)\right)_{i<j}$ : this follows from Eq.(3), by considering the quadrangle $1, i, j, k$. The latter sequence moreover appears as the inversion sequence of the permutation $w=1 w^{\prime}$ such that $V(w)=v$ (see the proof of Theorem 12).

\section{Properties of the poset}

We establish three properties of the poset of this article: it is a lattice, its covering degrees are related to Eulerian numbers, and its limiting poset is Young's lattice.

\section{$5.1 \quad$ Lattice}

Recall that the set of admitted vectors in $\mathbb{N}^{T}$ has been defined if the previous section.

Theorem 23. The poset of admitted vectors (or equivalently the poset of circular permutations) is a lattice.

We give a combinatorial proof of this result, with a simple algorithmic construction of the supremum and the infimum. This result may also be deduced from Theorem 36: see the comment before Corollary 44 .

We extend the definition of admitted vector as follows: if $v \in \mathbb{N}^{T}$, and $i<k$, then we say that the component $v_{i k}$ of $v$ is admitted if either $k=i+1$ and $v_{i k}=0$ or $k>i+1$ and

$$
\forall j, i<j<k \Rightarrow v_{i j}+v_{j k} \leqslant v_{i k} \leqslant v_{i j}+v_{j k}+1 .
$$

Hence $v$ is admitted if and only if all its components are admitted.

Lemma 24. If for $v \in \mathbb{N}^{T}$ all components $v_{i k}$ with $k-i<n-1$ are admitted, then the numbers $v_{1 i}+v_{\text {in }}, i=2, \ldots, n-1$ take at most two values, which are consecutive.

Proof. By hypothesis, we have for $1<i<j<n, v_{1 j}=v_{1 i}+v_{i j}+\eta_{1 i j}$ and $v_{i n}=$ $v_{i j}+v_{j n}+\eta_{i j n}$ for some $\eta_{1 i j}, \eta_{i j n} \in\{0,1\}$. It follows that

$$
v_{1 i}+\eta_{1 i j}+v_{i n}=v_{1 j}-v_{i j}+v_{i j}+v_{j n}+\eta_{i j n}=v_{1 j}+v_{j n}+\eta_{i j n} .
$$


Since the $\eta$ 's take only the values 0 or 1 , it follows that any two of the numbers $v_{1 i}+v_{i n}$, $i=2, \ldots, n-1$, differ in absolute value by at most 1 . It follows easily that they take at most two values, which must be consecutive.

By appropriately reindexing the vector, we obtain the following corollary.

Corollary 25. Suppose that $v \in \mathbb{N}^{T}$ and that, for some $i<j$, the components $v_{r s}$ with $s-r<j-i$ are admitted. Then the numbers $v_{i p}+v_{p j}, p=i+1, \ldots, j-1$, take at most two values, which are consecutive.

Proof of Theorem 23. Let $u, v$ be two admitted vectors. We define $X \in \mathbb{N}^{T}$ as follows: $X_{i j}$ is defined by induction on $j-i \geqslant 1$; if $j=i+1, X_{i j}=0$; if $j>i+1$, then $X_{i j}=\max \left\{u_{i j}, v_{i j}, X_{i p}+X_{p j}, i<p<j\right\}$.

1. We show first that $X$ is an admitted vector. It is enough to show that each coefficient $X_{i j}$ is admitted, and we do it by induction on $j-i$. The case $j-i=1$ is clear, so take $j-i=h>1$. By induction, all coefficients $X_{r s}$ with $s-r<h$ are admitted.

Suppose that $X_{i j}=X_{i p}+X_{p j}$ for some $p$ with $i<p<j$. By the corollary, one has $\left\{X_{i q}+X_{q j}, i<q<j\right\} \subset\{a-1, a\}$, and by the definition of $X_{i j}$, we must have $a=X_{i p}+X_{p j}$. It follows that for any $q, i<q<j$, one has $X_{i q}+X_{q j}=X_{i j}$ or $X_{i j}-1$ and therefore $X_{i q}+X_{q j} \leqslant X_{i j} \leqslant X_{i q}+X_{q j}+1$.

If $X_{i j}$ is not equal to $X_{i p}+X_{p j}$ for some $p$ with $i<p<j$, then we must have (choosing one of the two similar cases) $X_{i j}=v_{i j} \geqslant u_{i j}$ and $v_{i j}>X_{i p}+X_{p j}$ for any $p$ with $i<p<j$. Then $X_{i p}+X_{p j}<X_{i j}$; moreover $v$ being admitted, $X_{i j}=v_{i j} \leqslant v_{i p}+v_{p j}+1 \leqslant X_{i p}+X_{p j}+1$, since by the recursive construction of $X, v_{r s} \leqslant X_{r s}$ for any $r s$ with $s-r<j-i$.

We conclude that in all cases, $X_{i j}$ is admitted, and therefore by induction $X$ is admitted.

2. We show now that $X$ is the supremun of $u$ and $v$, that is, $X$ is the smallest element in the set of upper bounds of $u, v$. By construction, $X_{i j} \geqslant u_{i j}, v_{i j}$, so that $X \geqslant u, v$ and $X$ is an upper bound of $u$ and $v$. Now let $Y$ be an admitted vector which is an upper bound of $u, v$. We show by induction on $j-i$ that $X_{i j} \leqslant Y_{i j}$, which will imply that $X \leqslant Y$, as was to be shown. We have $X_{i j}=0=Y_{i j}$ if $j-i=1$. Suppose that $j-i>1$. Then $Y_{i j} \geqslant u_{i j}, v_{i j}$; and by the inequalities (2), if $i<p<j, Y_{i j} \geqslant Y_{i p}+Y_{p j}$ which by induction is $\geqslant X_{i p}+X_{p j}$; thus $Y_{i j} \geqslant \max \left\{u_{i j}, v_{i j}, X_{i p}+X_{p j}, i<p<j\right\}=X_{i j}$.

3. Thus $u \vee v$ exists in the poset. Since the poset has an anti-automorphism, $u \wedge v$ exists, too, and the poset is a lattice.

The proof allows to compute $u \vee v$. A direct computation of $X=u \wedge v$ is obtained recursively, by induction on $j-i$, as follows: $X_{i, i+1}=0$; if $j>i+1$, then $X_{i j}=$ $\min \left\{u_{i j}, v_{i j}, X_{i p}+X_{p j}+1, i<p<j\right\}$. The proof is left to the reader (one may use the first anti-automorphism of Corollary 19).

The lattice is not modular. Indeed, looking in Figure 2, it is seen that the circular permutations (14235) and (13425) have infimum (14253) and supremum (13542), and respective rank 4,4,3 and 6 . Since in a modular lattice, the rank function $\rho$ satisfies $\rho(x)+\rho(y)=\rho(x \wedge y)+\rho(x \vee y)$ (see [17] page 104), the lattice is not modular. Since each distributive lattice is modular ([17] p. 106), the lattice is not distributive either. 


\subsection{Multiplicities in the Hasse diagram and Eulerian numbers}

Recall that the Eulerian numbers are defined as follows: $a(n, k)$ is the number of permutations in $S_{n}$ whose descent set has $k$ elements, where the descent set of $\sigma \in S_{n}$ is $\{i, 1 \leqslant i \leqslant n-1, \sigma(i)>\sigma(i+1)\}$. These numbers satisfy the recursion

$$
a(n, k)=(k+1) a(n-1, k)+(n-k) a(n-1, k-1)
$$

if $n, k \geqslant 1$, with the initial conditions $a(0, k)=0$ for any $k \geqslant 1$, and $a(n, 0)=1$ for any $n \geqslant 0$ (see [12] for more details about Eulerian numbers).

Definition 26. Let $\sigma=\left(a_{1} \cdots a_{n}\right)$ be a circular permutation in $S_{n}$. We call large circular descent of $\sigma$ a letter $a_{i}$ such that $a_{i-1}>a_{i}+1$, were the indices are taken modulo $n$. The number of large circular descents of $\sigma$ is denoted by $d(\sigma)$.

Symmetrically, we call large circular ascent of $\sigma$ a letter $a_{i}$ such that $a_{i}<a_{i+1}+1$, were the indices are taken modulo $n$.

In other words, $b$ is a large circular descent if, denoting by $a$ the letter before $b$ in the cycle, one has $a>b+1$. Note that we say that $b$ is the large circular descent, and not $a$; the reason for this notational shift will appear in the proofs below. For example, the large circular descents of $(1,4,2,6,5,3)$ are 1 (because before 1 there is 3 , cyclically speaking), 2 (because before 2 there is 4 ), and $3 ; 5$ is not a large circular descent.

Theorem 27. The number of circular permutations in $S_{n+1}$ having $k$ large circular descents is equal to the Eulerian number $a(n, k)$.

One can note that the cover relations of the poset of circular permutations defined in Definition 3 correspond to the large circular descents defined in Definition 26. This gives us directly the following result.

Corollary 28. In the poset of circular permutations in $S_{n+1}$, the number of elements which are covered by $k$ elements is $a(n, k)$. In particular the number of inf-irreducible elements is $a(n, 1)=2^{n}-n-1$.

The next corollary is somewhat curious, since the two posets are not isomorphic, nor are their Hasse diagram as (undirected) graphs.

Corollary 29. The total number of edges in the Hasse diagram of the poset of circular permutations in $S_{n+1}$ is equal to the total number of edges in the Hasse diagram of the poset of the weak order in $S_{n}$.

Proof. This follows from the theorem since the edges in the Hasse diagram of the right weak order are in bijection with the set of descents of all permutations.

To prove the theorem, we show that the number of circular permutations in $S_{n+1}$ with $k$ large circular descents satisfy the same recursion as the Eulerian numbers, and the same initial conditions. Finding a bijective proof is an open question. 
Proof of Theorem 2\%. 1. Denote by $C(n)$ the set of circular permutations in $S_{n+1}$, so that $|C(n)|=n$ !. Denote by $C(n, k)$ the set of elements in $C(n)$ having $k$ large circular descents, and $c(n, k)=|C(n, k)|$.

We verify first that the initial conditions are the same. Indeed, $c(0, k)$ for $k \geqslant 1$ is the number of circular permutations in $S_{1}$ having $k$ large circular descents, hence $c(0, k)=0$. Moreover, for $n \geqslant 0, c(n, 0)$ is the number of circular permutations in $S_{n+1}$ having no large circular descent; this permutation is $(n, n-1, \ldots, 2,1)$ as we saw in the proof of Corollary 4 , and therefore $c(n, 0)=1$.

We show below that

$$
c(n, k)=(k+1) c(n-1, k)+(n-k) c(n-1, k-1),
$$

which is the same recursion as the Eulerian numbers. Thus we will obtain $c(n, k)=a(n, k)$, which proves the theorem.

2. Recall that each element in $C(n)$ is represented by a circular word $\left(a_{1}, \ldots, a_{n+1}\right)$ and that we do not distinguish between all its cyclic rearrangements (so that e.g. $\left(a_{1}, \ldots, a_{n+1}\right)$ $\left.=\left(a_{2}, \ldots, a_{n+1}, a_{1}\right)\right)$.

3. Define a mapping $\phi$ from $C(n)$ into $C(n-1) \times[n]$ as follows. Associate with $\alpha=\left(a_{1}, \ldots, a_{n+1}\right) \in C(n)$ the circular permutation $\beta=\left(b_{1}, \ldots, b_{n}\right) \in C(n-1)$ obtained by removing $n+1$ from the cycle, and let $b_{j}$ denote the letter such that $\left(a_{1}, \ldots, a_{n+1}\right)$ is obtained from $\beta=\left(b_{1}, \ldots, b_{n}\right)$ by inserting $n+1$ before $b_{j}$.

To illustrate the notions above we give the two following examples for $n+1=7$ :

- $\alpha_{1}=(2,4,6,5,1,7,3) \in C(6), \beta_{1}=(2,4,6,5,1,3) \in C(5), b_{j}=3 \in[6]$;

- $\alpha_{2}=(2,3,6,5,1,4,7), \beta_{2}=(2,3,6,5,1,4), b_{j}=2$.

Therefore, $\phi$ is defined by $\phi(\alpha)=\left(\beta, b_{j}\right)$. This is clearly a bijection, since we recover $\alpha$ from $\beta$ by inserting $n+1$ before $b_{j}$, so that $\phi$ is injective, and since the two sets have the same cardinality.

4. For $\beta \in C(n-1)$, define $D(\beta)$ to be the set of large circular descents of $\beta$; hence $|D(\beta)|=d(\beta)$ (where $d(\beta)$ is the number of large circular descents as defined in Definition 26).

5. Let $X:=X_{1} \sqcup X_{2}$ where $X_{1}$ and $X_{2}$ are defined by

$$
X_{1}:=\{(\beta, b), \beta \in C(n-1, k), b \in D(\beta) \cup\{n\}\},
$$

and

$$
X_{2}:=\{(\beta, b), \beta \in C(n-1, k-1), b \notin D(\beta) \cup\{n\}\} .
$$

We claim that the restriction of the mapping $\phi$ to $C(n, k)$ is a bijection from $C(n, k)$ onto $X$. It will follow that $c(n, k)=|X|$.

6. To prove the claim, it is enough to show that $\phi(C(n, k))=X$, since $\phi$ is bijective.

Consider $\alpha$ and $\beta$ satisfying $\phi(\alpha)=\left(\beta, b_{j}\right)$, with the notations in 3 . Then $\alpha=$ $\left(b_{1}, \ldots b_{j-1}, n+1, b_{j}, \ldots, b_{n}\right)$ and $\beta=\left(b_{1}, \ldots b_{j-1}, b_{j}, \ldots, b_{n}\right)$. We remark that: 
- If $b_{j} \in D(\beta)$ or if $b_{j}=n$, then $D(\alpha)=D(\beta)$ and $d(\alpha)=d(\beta)$.

- If $b_{j} \notin D(\beta)$ and $b_{j} \neq n$, then $D(\alpha)=D(\beta) \cup\left\{b_{j}\right\}$ and $d(\alpha)=d(\beta)+1$.

Note indeed that $n+1$ is not a large circular descent of $\alpha$. It follows that for $i \neq j, b_{i}$ is a large circular descent of $\alpha$ if and only $b_{i}$ is a large circular descent of $\beta$. Next, if $b_{j} \in D(\beta)$, then $b_{j} \in D(\alpha)$. Moreover, if $b_{j}=n$, then $b_{j}$ is neither in $D(\alpha)$ nor in $D(\beta)$. Finally, if $b_{j} \notin D(\beta)$ and $b_{j} \neq n$, then $b_{j} \in D(\alpha)$.

(i) We show that $\phi(C(n, k)) \subset X$. Suppose that $\alpha \in C(n, k)$.

If $b_{j} \in D(\beta)$ or if $b_{j}=n$, then by the remark, $\alpha$ and $\beta$ have the same number $k$ of large circular descents; hence $\left(\beta, b_{j}\right) \in X_{1}$. If on the contrary, $b_{j} \notin D(\beta)$ and $b_{j} \neq n$, then $\beta$ has $k-1$ large circular descents and thus $\left(\beta, b_{j}\right) \in X_{2}$.

(ii) We show now the inclusion $X \subset \phi(C(n, k))$. Suppose that $\left(\beta, b_{j}\right) \in X$. It is enough to show that $\alpha \in C(n, k)$.

If $\left(\beta, b_{j}\right) \in X_{1}$, then $d(\beta)=k$. Moreover, $b_{j} \in D(\beta)$ or $b_{j}=n$; thus by the remark, $\alpha$ and $\beta$ have the same number of large circular descents, hence $\alpha \in C(n, k)$.

If $\left(\beta, b_{j}\right) \in X_{2}$, then $d(\beta)=k-1$, and similarly, $\alpha$ has one large circular descent more than $\beta, \alpha \in C(n, k)$.

7. The set $C(n, k)$ has $c(n, k)$ elements. Moreover, by 5 , we know that $c(n, k)=|X|=$ $\left|X_{1}\right|+\left|X_{2}\right|$. However it is clear that $\left|X_{1}\right|=c(n-1, k)(k+1)$ and $\left|X_{2}\right|=c(n-1, k-1)(n-k)$. Thus the recursion follows.

\subsection{Limiting poset}

In this section, we show that the limit (in some precise sense) of the posets of circular permutations in $S_{n}$ is, when $n$ tends to infinity, Young's lattice of partitions.

Suppose that we have a family $P_{n}, n \in \mathbb{N}$, of ranked posets; suppose also that for any $k$, and for $n$ large enough, the subposets $\left\{x \in P_{n}, r k(x) \leqslant k\right\}$ are all isomorphic. We may then define the limit of these posets as the ranked poset $P$, whose elements of rank $\leqslant k$ form the poset $\left\{x \in P_{n}, r k(x) \leqslant k\right\}, n$ large enough.

Recall that a partition of $n$ is a multiset of positive integers whose sum is $n$ ( $n$ is the weight of the partition); a partition of $n+1$ covers a partition of $n$ if the latter is obtained from the latter by adding 1 to the multiset, or by replacing some element $a$ by $a+1$. This is Young's lattice, with rank equal to the weight function; see [15] Definition 5.1.2.

Let us also recall how the poset of circular permutations is constructed. First we represent the smallest element (which is the canonical $n$-cycle) by $\left(w_{0}\right)$, where $w_{0}$ is the word

$$
w_{0}:=u_{0} v_{0}=(\lfloor n / 2\rfloor+1) \ldots(n-1) n 12 \ldots\lfloor n / 2\rfloor .
$$

For example, in the cases $n=5,6$, it is respectively 34512 and 456123 .

Next each circular permutation is obtained, starting from $\left(w_{0}\right)$, by applying iteratively the covering relation $(w) \rightarrow\left(w^{\prime}\right)$, defined by

$$
w=u s r v, w^{\prime}=u r s v \text { or } w=r u s, w^{\prime}=s u r, s>r+1 .
$$


In the first case we say that the covering relation is inner, and the second one, it is outer. In this way, we obtain for each circular permutation a unique representative $(w)$, as follows from Section 2.

Lemma 30. Let $(w) \in S_{n}$ be a circular permutation, represented as above. Then the following conditions are equivalent:

i) We can obtain $(w)$ from $\left(w_{0}\right)$ by a sequence of inner covering relations.

ii) The word $w$ is a shuffle of $u_{0}$ and $v_{0}$.

Example 31. Let $n=8$ and $w=56172384$. Then, using inner relations from $w_{0}$ we get:

$$
56781234 \rightarrow 56718234 \rightarrow 56178234 \rightarrow 56172834 \rightarrow 56172384
$$

Proof. i) $\Rightarrow$ ii). We proceed by induction on the number of inner relations $p$. For $p=0$ there is nothing to do because $w=w_{0}$. Assume that $w$ is obtained from $w_{0}$ by $p+1$ inner relations. Then we can write $w=u r s v$ where $r, s$ are letters such that $r+1<s$ (that is swapping $r$ and $s$ is the last inner relation to obtain $w)$. Let $w^{\prime}:=u s r v$. This word is obtained from $w_{0}$ using only $p$ inner relations. Then by induction $w^{\prime}$ is a shuffle of $u_{0}$ and $v_{0}$. Therefore, if $s, r$ are simultaneously in $u_{0}$ or $v_{0}$, they are consecutive and we must have $s=r-1$. This is in contradiction with our assumption. Thus $s$ is a letter of $u_{0}$ and $r$ is a letter of $v_{0}$. Swapping $s$ and $r$ does not change appearance order of the letters of both $u_{0}$ and $v_{0}$. Therefore, $w$ is also a shuffle of $u_{0}$ and $v_{0}$. This ends the first direction.

The direction ii) $\Rightarrow$ i) is obvious.

Theorem 32. The posets of circular permutations have a limit poset, which is Young's lattice.

Proof. 1. We prove first that the limit exists. Let $k$ be an integer. Suppose that $n \geqslant 2 k$. We show that the subposet of elements of rank $\leqslant k$ is independent of $n$.

Let $(x)$ be a circular permutation of $S_{n}$ such that $r k(x) \leqslant k$. Then $x$ is obtained from $w_{0}$ using at most $k$ covering relations. Moreover, since the first covering relation is necessarily determined by the middle factor $n 1$, and since $k \leqslant\lfloor n / 2\rfloor$, we never perform an outer covering relation. This implies that the subposet of elements of rank $\leqslant k$ is independent of $n$.

2. To finish the proof, it is enough to prove that if $n \geqslant 2 k$, then the subposet of elements of rank $\leqslant k$ is isomorphic with the subposet of the Young lattice whose elements are the partitions of weight $\leqslant k$.

By the previous part and because of Lemma 30, each element of rank $\leqslant k$ is of the form $(w)$, where $w$ a shuffle of the two words $u_{0}$ and $v_{0}$. Furthermore, the order is the inverse order of the right weak order on these words.

Note that each such shuffle, $w$ say, is determined by the sequence $a_{1}, a_{2}, a_{3}, \ldots, a_{\lfloor n / 2\rfloor}$, where $a_{i}$ is the number of $j=(\lfloor n / 2\rfloor+1), \ldots,(n-1), n$, which are at the right of $i$ in $w$. The rank of $w$ is the sum of the $a_{i}$ 's. One has $a_{1} \geqslant a_{2} \geqslant a_{3} \ldots$, and therefore the sequence determines a partition of some integer $\leqslant k$. This sequence defines the isomorphism between the two posets. We leave the details to the reader. 


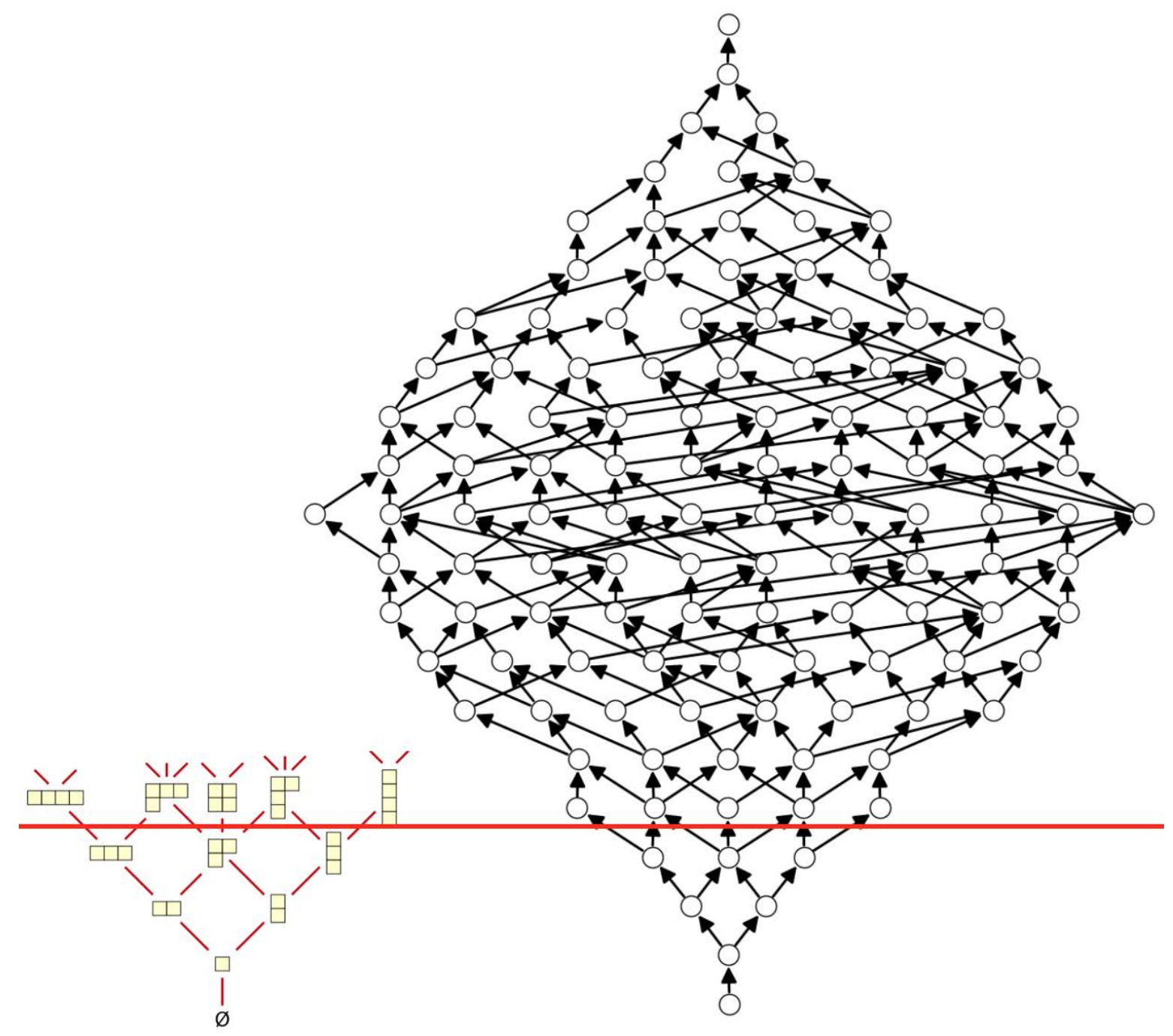

Figure 7: Illustration of the proof of Theorem 32. On the left hand side, the beginning of Young's lattice, and the right hand side, the circular permutations lattice for $n=6$.

Example 33. We continue Example 31. Let $w=56172384$. Then $a_{1}$ is the number of blue letters which are on the right side of 1 . That is $a_{1}=2$. Proceeding this way for all other cases we get $a_{2}=1, a_{3}=1, a_{4}=0$. Thus, the partition corresponding to $w$ is $2,1,1,0$.

\section{An interval in the affine symmetric group}

The two isomorphic posets of circular permutations and admitted vectors are isomorphic to some interval of the affine symmetric group, ordered by the left weak order. Thus we turn back to the geometric motivations of this article, following the second author's articles $[5,6]$. 


\subsection{Preliminaries on $\tilde{S}_{n}$}

We begin by recalling some definitions and results on the affine symmetric group. Details may be found in the book by Anders Björner and Francesco Brenti [4]. This group is denoted $\tilde{S}_{n}$. It is the group of permutations $f$ of $\mathbb{Z}$ satisfying the two conditions:

- $f(x+n)=f(x)+n$ for any integer $x$;

- $f(1)+f(2)+\cdots+f(n)=n(n+1) / 2$.

An element of $\tilde{S}_{n}$ is called an affine permutation. Each usual permutation in $S_{n}$ extends uniquely to an affine permutation, so that $\tilde{S}_{n}$ contains the symmetric group $S_{n}$ as a subgroup. The group $\tilde{S}_{n}$ is generated by the adjacent affine transpositions $s_{0}, s_{1}, \ldots, s_{n-1}$, defined by the condition that $s_{i}$ exchanges $i$ and $i+1$ if $i=0, \ldots, n-1$. Each affine permutation $f$ is represented by a window $[f(1), f(2), \ldots, f(n)]$ and we write $f=[f(1), f(2), \ldots, f(n)]$. For example in $\tilde{S}_{4}$, we have the elements $s_{1}=[2,1,3,4]$, $s_{0}=[0,2,3,5]$, and $[-3,5,0,8]$. The $n$ numbers in a window are pairwise noncongruent modulo $n$, and their sum is $n(n+1) / 2$; windows are characterized by these two previous properties.

The position inversion set of $f \in \tilde{S}_{n}$ is the set

$$
\operatorname{Inv}_{p}(f):=\{(i, j) \mid 1 \leqslant i \leqslant n, i<j, f(i)>f(j)\},
$$

which is also equal to

$$
\{(i, j) \mid 1 \leqslant i \leqslant n, i<j, f(i) \geqslant f(j)\},
$$

since $i<j$ implies $f(i) \neq f(j)$.

The affine symmetric group is a Coxeter group, generated by the $s_{i}$ 's, with length function $\ell(f)=\left|\operatorname{Inv}_{p}(f)\right|$. The left weak order, denoted simply $\leqslant$, is defined on $\tilde{S}_{n}$ by its covering relation: $g$ covers $f$ if for some $i$, one has $g=s_{i} f$ and $\ell(g)=\ell(f)+1$.

By definition of the product in $\tilde{S}_{n}$, one has the following lemma (a variant of [4] p. 260).

Lemma 34. Let $k=0, \ldots, n-1$ and $\left[a_{1}, \ldots, a_{n}\right] \in \tilde{S}_{n}$. Define $i, j$ in $\{1,2, \ldots, n\}$ by $a_{i} \equiv k+1 \bmod n$ and $a_{j} \equiv k \bmod n$. Then $s_{k}\left[a_{1}, \ldots, a_{n}\right]=\left[b_{1}, \ldots, b_{n}\right]$, with $b_{j}=a_{j}+1$, $b_{i}=a_{i}-1$, and $b_{l}=a_{l}$ if $a_{l}$ is noncongruent to $k$ nor to $k+1$ modulo $n$.

We also need the following result, which follows from Theorem 16 in [7] (see also [3] Theorem 5.3).

Lemma 35. Let $f, g \in \tilde{S}_{n}$ and $k=0, \ldots, n-1$. Then:

(i) the sets $\operatorname{Inv}_{p}(f)$ and $\operatorname{Inv} v_{p}\left(s_{k} f\right)$ differ by one element;

(ii) $f \leqslant g$ if and only if $\operatorname{Inv} v_{p}(f) \subset \operatorname{Inv} v_{p}(g)$. 


\subsection{A poset isomorphism}

We define a mapping $F$ from the set of admitted vectors into $\mathbb{Z}^{n}$, where elements of the latter set are denoted by brackets $\left[a_{1}, \ldots, a_{n}\right]$. Let $v$ be an admitted vector. Then we define $F(v)=\left[a_{1}, \ldots, a_{n}\right]$, by

$$
a_{i}=i+\sum_{p<i} v_{p i}-\sum_{i<p} v_{i p}
$$

If we denote by $e_{1}, \ldots, e_{n}$ the canonical basis of $\mathbb{Z}^{n}$, we have equivalently

$$
F(v)=[1,2, \ldots, n]+\sum_{r<s} v_{r s}\left(e_{s}-e_{r}\right) .
$$

Now, consider the element $f_{c} \in \tilde{S}_{n}$ defined by its window: $f_{c}=\left[c_{1}, c_{2}, \ldots, c_{n}\right]$ with $c_{1}=-(1 / 2) n(n-3)$ and $c_{i+1}=c_{i}+n-1$ for $i=1, \ldots, n-1$. Since $n-1$ is a generator of the additive group of integers modulo $n$, the $c_{i}$ are pairwise distinct modulo $n$. Moreover the sum of all $c_{i}$ is

$$
\begin{aligned}
& \left(c_{1}+0\right)+\left(c_{1}+n-1\right)+\left(c_{1}+2(n-1)\right)+\cdots+\left(c_{1}+(n-1)(n-1)\right) \\
= & n c_{1}+\frac{(n-1) n(n-1)}{2} \\
= & -\frac{1}{2} n^{2}(n-3)+\frac{1}{2}(n-1)^{2} n \\
= & \frac{1}{2} n\left((n-1)^{2}-n(n-3)\right) \\
= & \frac{1}{2} n\left(n^{2}-2 n+1-n^{2}+3 n\right) \\
= & \frac{n(n+1)}{2} .
\end{aligned}
$$

This shows that indeed $f_{c} \in \tilde{S}_{n}$.

As an example, for $n=4, f_{c}=[-2,1,4,7]$ and for $n=5, f_{c}=[-5,-1,3,7,11]$.

Theorem 36. The mapping $F$ is a poset isomorphism from the set of admitted vectors onto the interval $\left[\mathrm{id}, f_{c}\right]$ with the left weak order.

Notice that this result has been proven by the second-named author for any affine Weyl group $W_{a}$ in [5] by using a geometrical approach. We give here a combinatorial proof and further, we construct the element $f_{c}$.

See Figure 8 for this interval. The cover relation is explained in Remark 39. For example (24). $[-1,1,3,5,7]=[-1,1-1,3,5+1,7]=[-1,0,3,6,7]$.

We first need several preliminary results.

Lemma 37. Let $F(v)=\left[a_{1}, \ldots, a_{n}\right]$ and $1 \leqslant i<j \leqslant n$. Then

$$
a_{j}-a_{i}=j-i+n v_{i j}-\sum_{i<p<j} \delta_{i p j}(v)+\sum_{p<i} \delta_{p i j}(v)+\sum_{j<p} \delta_{i j p}(v) .
$$

As a consequence, $a_{j}-a_{i}=n v_{i j}+r$, with $r \in\{1, \ldots, n-1\}$ and $\left\lfloor\frac{a_{j}-a_{i}}{n}\right\rfloor=v_{i j}$. 
Proof. We have

$$
\begin{aligned}
& a_{j}-a_{i} \\
= & j-i+\sum_{p<j} v_{p j}-\sum_{j<p} v_{j p}-\sum_{p<i} v_{p i}+\sum_{i<p} v_{i p} \\
= & j-i+\sum_{p<i} v_{p j}+v_{i j}+\sum_{i<p<j} v_{p j}-\sum_{j<p} v_{j p}-\sum_{p<i} v_{p i}+\sum_{i<p<j} v_{i p}+v_{i j}+\sum_{j<p} v_{i p} \\
= & j-i+2 v_{i j}+\sum_{i<p<j}\left(v_{i p}+v_{p j}\right)+\sum_{p<i}\left(v_{p j}-v_{p i}\right)+\sum_{j<p}\left(v_{i p}-v_{j p}\right) \\
= & j-i+2 v_{i j}+\sum_{i<p<j}\left(v_{i j}-\delta_{i p j}(v)\right)+\sum_{p<i}\left(v_{i j}+\delta_{p i j}(v)\right)+\sum_{j<p}\left(v_{i j}+\delta_{i j p}(v)\right) \\
= & j-i+n v_{i j}-\sum_{i<p<j} \delta_{i p j}(v)+\sum_{p<i} \delta_{p i j}(v)+\sum_{j<p} \delta_{i j p}(v) .
\end{aligned}
$$

Let $r=j-i-\sum_{i<p<j} \delta_{i p j}(v)+\sum_{p<i} \delta_{p i j}(v)+\sum_{j<p} \delta_{i j p}(v)$. Then

$$
a_{j}-a_{i}=n v_{i j}+r
$$

Moreover, since the $\delta$ 's take the values 0 and $1, r$ is in the interval $[j-i-(j-i-1), j-$ $i+i-1+n-j]=[1, n-1]$.

Proposition 38. The image of $F$ is contained in the set of windows. If $F(v)=\left[a_{1}, \ldots, a_{n}\right]$, then $a_{1}<\cdots<a_{n}$.

Proof. For the first assertion, it is enough to show that the $a_{i}$ as defined above are pairwise noncongruent modulo $n$, and that their sum is $n(n+1) / 2$.

Let $i<j$. Because of Lemma 37 we already know that $a_{i}-a_{j}$ is nonzero modulo $n$. Further we have

$$
\begin{aligned}
\sum_{i=1}^{n} a_{i} & =\sum_{i=1}^{n}\left(i+\sum_{p<i} v_{p i}-\sum_{i<p} v_{i p}\right) \\
& =\sum_{i=1}^{n} i+\sum_{p<i} v_{p i}-\sum_{i<p} v_{i p} \\
& =\sum_{i=1}^{n} i \\
& =\frac{n(n+1)}{2} .
\end{aligned}
$$

For the last assertion, let $1 \leqslant i<j \leqslant n$. By Lemma 37, $a_{j}-a_{i}>0$.

Remark 39. In this paragraph we recall how the cover relation works for an affine permutation $f$ (see Lemma 34). More precisely we give the cover relation in terms of the 
window corresponding to $f$. Let $g$ be another affine permutation such that $g$ covers $f$ and let $(i, j)$ be the transposition such that $(i, j) \cdot f=g$. Then

$$
(i, j) \cdot\left[a_{1}, \ldots, a_{i}, \ldots, a_{j}, \ldots, a_{n}\right]=\left[a_{1}, \ldots, a_{i}-1, \ldots, a_{j}+1, \ldots, a_{n}\right] .
$$

Lemma 40. Suppose that $f, g \in \tilde{S}_{n}$ with $f=\left[a_{1}, a_{2}, \ldots, a_{n}\right], g=\left[b_{1}, b_{2}, \ldots, b_{n}\right], a_{1} \leqslant$ $\cdots \leqslant a_{n}$ and $b_{1} \leqslant \cdots \leqslant b_{n}$. Then:

(i) $\operatorname{Inv}_{p}(f)$ is the set of pairs $(i, p+k n)$ with $1 \leqslant p<i \leqslant n$ and $k=1, \ldots,\left\lfloor\frac{a_{i}-a_{p}}{n}\right\rfloor$,

(ii) $f \leqslant g$ if and only if for any $i<j,\left\lfloor\frac{a_{j}-a_{i}}{n}\right\rfloor \leqslant\left\lfloor\frac{b_{j}-b_{i}}{n}\right\rfloor$.

Note that assertion (ii) is a particular case of Theorem 16 (3) in [7].

Proof. (i). A pair as in the statement is an inversion, since $i<p+k n$ and $f(i)=$ $a_{i} \geqslant f(p+k n)=f(p)+k n=a_{p}+k n$ because $k \leqslant\left\lfloor\frac{a_{i}-a_{p}}{n}\right\rfloor \leqslant \frac{a_{i}-a_{p}}{n}$. Conversely, let $(i, j)$ be an inversion of $f$. Then $1 \leqslant i \leqslant n$. Since $j>i \geqslant 1$, we may write $j=p+k n, 1 \leqslant p \leqslant n, k \geqslant 0$. We have $f(i)>f(j)=f(p)+k n$. We cannot have $p \geqslant i$, since otherwise we would have, by the increasing property, $f(i) \leqslant f(p) \leqslant f(j)$, a contradiction. Hence $p<i$ and since $p+k n=j>i$, we must have $k \geqslant 1$. Moreover, $k n<f(i)-f(p)=a_{i}-a_{p}$, hence $k \leqslant\left\lfloor\frac{a_{i}-a_{p}}{n}\right\rfloor$.

(ii) follows from (i).

Lemma 41. Consider two admitted vectors $v, v^{\prime}$ and $1 \leqslant i<j \leqslant n$ such that $v_{i j}^{\prime}=v_{i j}+1$ whereas $v_{r s}^{\prime}=v_{r s}$ for the other coordinates. Let $k=a_{j} \bmod n$, where $a_{j}$ is the $j$-th coordinate of $F(v)$. Then in $\tilde{S}_{n}$, one has the equality $F\left(v^{\prime}\right)=s_{k} F(v)$ and $\ell\left(F\left(v^{\prime}\right)\right)=$ $\ell(F(v))+1$.

Proof. Let $F(v)=\left[a_{1}, \ldots, a_{n}\right]$ and $F\left(v^{\prime}\right)=\left[a_{1}^{\prime}, \ldots, a_{n}^{\prime}\right]$. Then by Eq.(6):

$$
a_{i}^{\prime}=i+\sum_{p<i} v_{p i}^{\prime}-\sum_{i<p} v_{i p}^{\prime}=i+\sum_{p<i} v_{p i}-\sum_{i<p} v_{i p}-1=a_{i}-1,
$$

and similarly $a_{j}^{\prime}=a_{j}+1, a_{l}^{\prime}=a_{l}$ if $l \neq i, j$. By Lemma 37 and Lemma 14, $a_{j}-a_{i}$ is equal to $j-i+(i-1)+(n-j)=n-1 \equiv-1 \bmod n$. Hence $a_{i} \equiv k+1 \bmod n$.

Let $s_{k} F(v)=\left[b_{1}, \ldots, b_{n}\right]$. By Lemma 34, we deduce that $b_{i}=a_{i}-1$ and $b_{j}=a_{j}+1$, whereas $b_{l}=a_{l}$ for the other $l$. Hence $b_{l}=a_{l}^{\prime}$ for all $l$, that is, $F\left(v^{\prime}\right)=s_{k} F(v)$.

We have $a_{j}=a_{i}+n-1$. Hence

$$
F(v)=\left[\ldots, a_{i}, \ldots, a_{j}, \ldots\right]=\left[\ldots, a_{i}, \ldots, a_{i}+n-1, \ldots\right],
$$

and

$$
\begin{aligned}
F\left(v^{\prime}\right) & =\left[\ldots, a_{i}^{\prime}, \ldots, a_{j}^{\prime}, \ldots\right]=\left[\ldots, a_{i}-1, \ldots, a_{j}+1, \ldots\right] \\
& =\left[\ldots, a_{i}-1, \ldots, a_{i}+n, \ldots\right] .
\end{aligned}
$$

Thus $F\left(v^{\prime}\right)$ has the position inversion $(j, i+n)$ (because $F\left(v^{\prime}\right)$ send this pair onto $\left(a_{i}+\right.$ $\left.n, a_{i}+n-1\right)$ ), but $F(v)$ has not this inversion (because the pair $(j, i+n)$ is sent by $F(v)$ onto $\left.\left(a_{i}+n-1, a_{i}+n\right)\right)$. It follows from Lemma 35 (i) that the length of $F\left(v^{\prime}\right)$ is one more than the length of $F(v)$. 
$[-5,-1,3,7,11]$

(15)

$[-4,-1,3,7,10]$

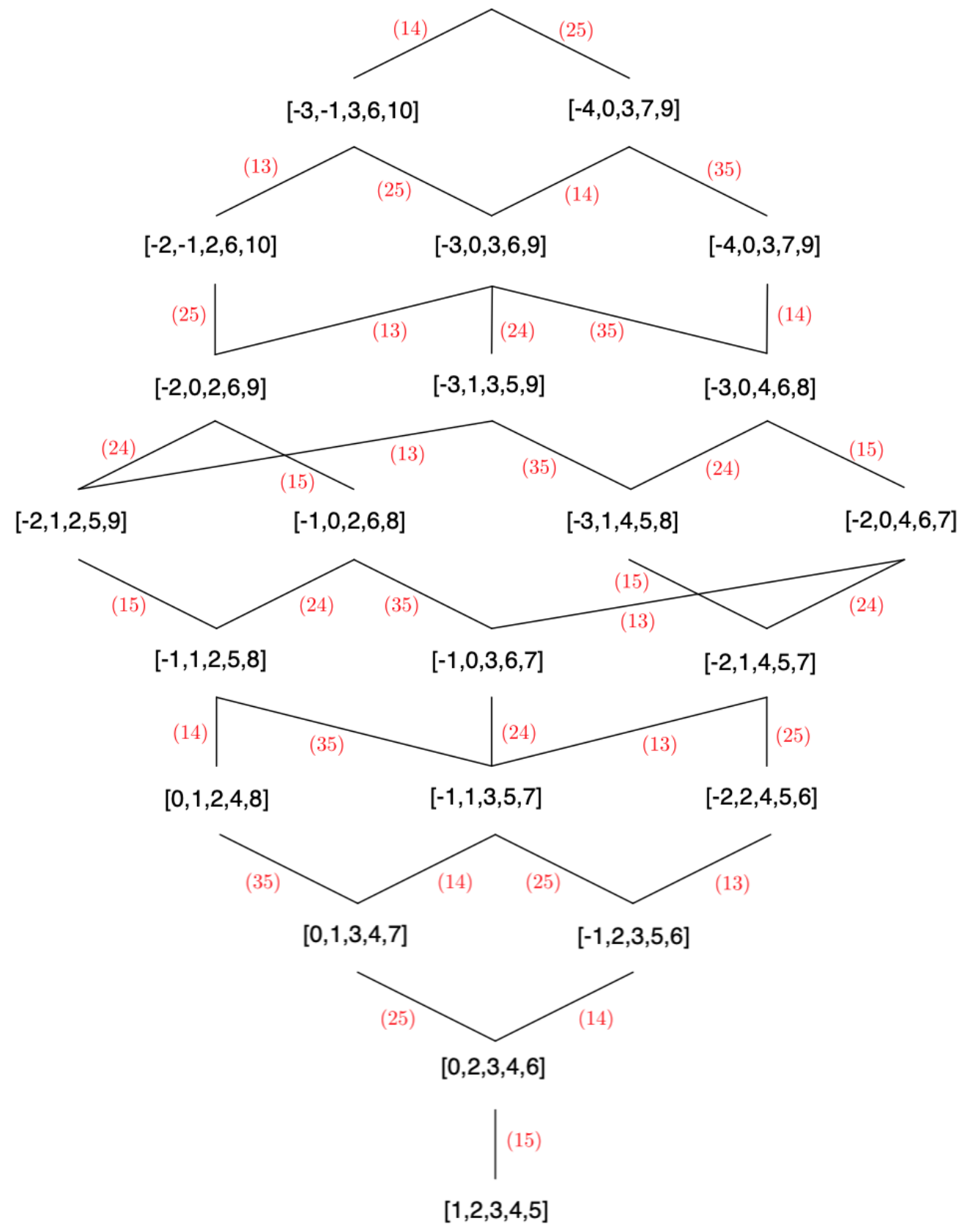

Figure 8: The interval in the affine symmetric group $\tilde{S}_{5}$. 
Lemma 42. Let $\left[a_{1}, \ldots, a_{n}\right] \in \tilde{S}_{n}$. Then

$$
a_{i}=i-\sum_{i<p}\left\lfloor\frac{a_{p}-a_{i}}{n}\right\rfloor+\sum_{p<i}\left\lfloor\frac{a_{i}-a_{p}}{n}\right\rfloor .
$$

This formula could be proved as a corollary of [16] Theorem 4.1, but we give a direct proof.

Proof. By definition of $\tilde{S}_{n}$, there exists a permutation $\sigma \in S_{n}$ and integers $b_{1}, \ldots, b_{n}$ such that $a_{i}=\sigma(i)+n b_{i}$ and $b_{1}+\cdots+b_{n}=0$. One has

$$
\begin{aligned}
A & :=i-\sum_{i<p}\left\lfloor\frac{a_{p}-a_{i}}{n}\right\rfloor+\sum_{p<i}\left\lfloor\frac{a_{i}-a_{p}}{n}\right\rfloor \\
& =i-\sum_{i<p}\left\lfloor\frac{\sigma(p)+n b_{p}-\sigma(i)-n b_{i}}{n}\right\rfloor+\sum_{p<i}\left\lfloor\frac{\sigma(i)+n b_{i}-\sigma(p)-n b_{p}}{n}\right\rfloor .
\end{aligned}
$$

Note that for integers $b, s,\left\lfloor\frac{n b+s}{n}\right\rfloor=b+\left\lfloor\frac{s}{n}\right\rfloor$, that $-1<\frac{\sigma(j)-\sigma(k)}{n}<1$, and that $\sigma(j)-\sigma(k)$ has integer part 0 or -1 depending on $\sigma(j)-\sigma(k) \geqslant 0$ or $<0$. Thus

$$
A=i-\sum_{i<p}\left(b_{p}-b_{i}\right)+\sum_{p<i}\left(b_{i}-b_{p}\right)-\sum_{i<p, \sigma(p)<\sigma(i)}(-1)+\sum_{p<i, \sigma(i)<\sigma(p)}(-1) .
$$

We claim that $\sum_{i<p, \sigma(p)<\sigma(i)} 1=\sigma(i)-i+\sum_{p<i, \sigma(i)<\sigma(p)} 1$. It follows that

$$
A=i+(n-1) b_{i}-\sum_{p \neq i} b_{p}+\sigma(i)-i=\sigma(i)+n b_{i}=a_{i}
$$

because $-\sum_{p \neq i} b_{p}=b_{i}$.

In order to prove the claim, note that since $\sigma$ is a permutation, one has for fixed $i$ :

$$
\sum_{i<p, \sigma(p)<\sigma(i)} 1+\sum_{i>p, \sigma(p)<\sigma(i)} 1=\sum_{\sigma(p)<\sigma(i)} 1=\sigma(i)-1,
$$

and

$$
\sum_{i>p, \sigma(p)<\sigma(i)} 1+\sum_{i>p, \sigma(p)>\sigma(i)} 1=\sum_{i>p} 1=i-1
$$

Hence

$$
\sum_{i<p, \sigma(p)<\sigma(i)} 1=\sigma(i)-1-\sum_{i>p, \sigma(p)<\sigma(i)} 1=\sigma(i)-1-\left(i-1-\sum_{i>p, \sigma(p)>\sigma(i)} 1\right) .
$$

We prove now Theorem 36. The mapping $G=F^{-1}$, defined in the proof, appears implicitly in [7] p.20 and Eq. (4.2.1) p. 375 in [17]. 
Proof of Theorem 36. 1. It is easy to see that $F$ sends the null admitted vector onto id $=[1,2, \ldots, n]$. We show that $F(w)=f_{c}$, where $w$ is the largest admitted vector, so that $w_{i j}=j-i-1$ (Corollary 20). Let $F(w)=\left[a_{1}, \ldots, a_{n}\right]$. Then

$$
\begin{aligned}
a_{1}=1-\sum_{1<p \leqslant n} w_{1 p} & =1-\sum_{1<p \leqslant n}(p-2)=1-\frac{(n-1)(n-2)}{2} \\
& =\frac{2-n^{2}+3 n-2}{2} \\
& =-\frac{n(n-3)}{2} .
\end{aligned}
$$

Moreover, by Lemma 37, noting that

$$
a_{i+1}-a_{i}=1+\sum_{p<i} \delta_{p, i, i+1}(w)+\sum_{i+1<p} \delta_{i, i+1, p}(w) \quad \text { and } \quad v_{i, i+1}=0
$$

we have by Corollary 20 that it is equal to $1+(i-1)+(n-i-1)=n-1$. It follows that $a_{i}=c_{i}$, and $F(w)=f_{c}$.

2. Let $v$ be an admitted vector and $F(v)=f$. We have $v_{i j} \leqslant w_{i j}$, so that by Proposition 38, Lemma 40 (ii) and Lemma 37, one has $f \leqslant f_{c}$. By the same results, id $\leqslant f$. Thus $f \in\left[\right.$ id, $\left.f_{c}\right]$.

3. Let $0 \leqslant i<j \leqslant n$. The injectivity of $F$ follows from the equality $v_{i j}=\left\lfloor\frac{a_{j}-a_{i}}{n}\right\rfloor$, see Lemma 37.

4. Given $f \in\left[\mathrm{id}, f_{c}\right]$, define $G(f)=v \in \mathbb{Z}^{T}$ by $v_{i j}=\left\lfloor\frac{a_{j}-a_{i}}{n}\right\rfloor$. The previous paragraph shows that $G \circ F=$ id.

We show that the image of $G$ is contained in the set of admitted vectors. For this note that for an integer $x,\lfloor x / n\rfloor$ is the quotient of the Euclidean division of $x$ by $n$; therefore $\lfloor(x+y) / n\rfloor=\lfloor x / n\rfloor+\lfloor y / n\rfloor+\epsilon$, with $\epsilon=0$ or 1 . It follows that if $i<j<k$, $\left\lfloor\frac{a_{k}-a_{i}}{n}\right\rfloor-\left\lfloor\frac{a_{k}-a_{j}}{n}\right\rfloor-\left\lfloor\frac{a_{j}-a_{i}}{n}\right\rfloor$ is equal to 0 or 1 , and $v_{i j}$ is thus an admitted vector.

Observe that $f=\left[a_{1}, \ldots, a_{n}\right] \in \tilde{S}_{n}$ has no inversion of the form $(i, j)$ with $1 \leqslant i<$ $j \leqslant n$ if and only if $a_{1}<\ldots<a_{n}$. This is the case in particular for $f=f_{c}$. Therefore each $f \in\left[\mathrm{id}, f_{c}\right]$ satisfies this increasing condition, because $f \leqslant f_{c}$ implies that by Lemma 35 , each inversion of $f$ is an inversion of $f_{c}$. The nonnegativity of the coefficients of $G(f)$ follows.

5. The equality $F \circ G=$ id follows from Lemma 42 .

6. It remains to show that $F$ and $G$ are increasing functions. That $F$ is increasing follows from Lemma 41 and the description in Lemma 15 of the covering relation for admitted vectors.

Suppose now that $f, g \in\left[\mathrm{id}, f_{c}\right]$ and $f \leqslant g$. Then $f, g$ satisfy the hypothesis of Lemma 40 and it follows by (ii) in this lemma that $G(f) \leqslant G(g)$.

By composing the bijections, we obtain a direct poset isomorphism from the interval $\left[\mathrm{id}, f_{c}\right]$ into the set of circular permutations. Recall that $\tilde{S}_{n}$ is the semidirect product of $S_{n}$ and of the additive group $\left\{\left(k_{1}, \ldots, k_{n}\right) \in \mathbb{Z}^{n}, \sum_{i} k_{i}=0\right\}$. The canonical projection 
$\tilde{S}_{n} \rightarrow S_{n}$ is defined by $f \mapsto \sigma$, with $\sigma(i)=f(i) \operatorname{MOD} n$, where $x \operatorname{MOD} n$ is the unique representative modulo $n$ of $x$ in $\{1, \ldots, n\}$.

Corollary 43. The poset isomorphism $V^{-1} \circ F^{-1}$ from [id, $f_{c}$ ] into the set of circular permutations in $S_{n}$ is defined by $f \mapsto(\sigma)$, where $\sigma$ is as above.

Proof. This follows from the definition of $G$ and of the proof of Proposition 9 at the end of Section 3.

In a Coxeter group, an interval $I$ is always a lattice. Indeed it is well known that the right weak order is a complete meet-semilattice (see [4] Section 3.2). Therefore each pair of elements $x, y \in I$ has an infimum. Since $I$ admits a upper bound it turns out that each pair of elements $x, y \in I$ has also a supremum. Further, it is known that such an interval is a semidistributive lattice (see [13] Theorem 8.1 and the comment after it). Consequently, the poset considered in the present article has the following property.

Corollary 44. The poset of circular permutations in $S_{n}$ is a semidistributive lattice.

We leave to the reader to verify that $f_{c}$ is an involution in $\tilde{S}_{n}$ if $n$ is odd, but not when $n$ is even.

Define the elements $s_{i j k}$ of $\tilde{S}_{n}$, where $1 \leqslant i<j \leqslant n$ and $k \in \mathbb{Z}$ for any $x \in\{1,2, \ldots, n\}$ by

$$
s_{i j k}(x)=\left\{\begin{array}{ccc}
j-k n & \text { if } & x=i \\
i+k n & \text { if } & x=j \\
x & \text { if } & x \neq i, j
\end{array}\right.
$$

Proposition 45. Let $v$ be an admitted vector and $f=F(v)$. Consider the upwards path, in the Hasse diagram of the poset of admitted vectors, from the null vector to $v$, with successive labels $\left(i_{1}, j_{1}\right), \ldots,\left(i_{r}, j_{r}\right)$, where $r$ is the rank of $v$ in the poset. For $p=1, \ldots, r$, let $k_{p}$ be the number of $q \leqslant p$ such that $\left(i_{q}, j_{q}\right)=\left(i_{p}, j_{p}\right)$. Then in $\tilde{S}_{n}$, one has the factorization

$$
f=s_{i_{1} j_{1} k_{1}} \circ \cdots \circ s_{i_{r} j_{r} k_{r}} .
$$

The proof is left to the reader.

Note that it follows from Theorem 36, and from Corollary 3.2.8 in [4], that the Möbius function of the poset takes its values in $\{-1,0,1\}$.

\section{Circular line diagrams}

In this section, we introduce circular line diagrams. They are inspired by classical line diagrams for permutations, but instead of being on a flat plane, they are drawn on a cylinder, allowing us to use the circular properties of circular permutations. We will see that if such diagram respects certain properties on its crossings, it links a circular permutation $\sigma$ to its associated vector $V(\sigma)$ (see Corollary 48). Thus, we illustrate the bijection $V$ with a nice geometric and combinatorial object. 
We define a circular line diagram $D$ as follows: we take a cylinder, and we put in clockwise order the numbers from 1 to $n$ at the top and in any order at the bottom. For any $1 \leqslant i \leqslant n$, we draw a continuous oriented line that goes from $i$ at the top to $i$ at the bottom, and does not cross itself.

We can represent this diagram on a 2-dimensional surface by either projecting it like in Figure 9, or unrolling it like in Figure 10.

By turning clockwise around the cylinder, we can list the numbers at the bottom starting with 1 and we obtain a word $w=1 i_{1} \cdots i_{n-1}$. We then say that a circular line diagram $D$ represents the circular permutation $(w)$. For example, in Figures 9 and 10 below, we draw two circular line diagrams representing the same permutation $(1,6,4,2,3,5)$.

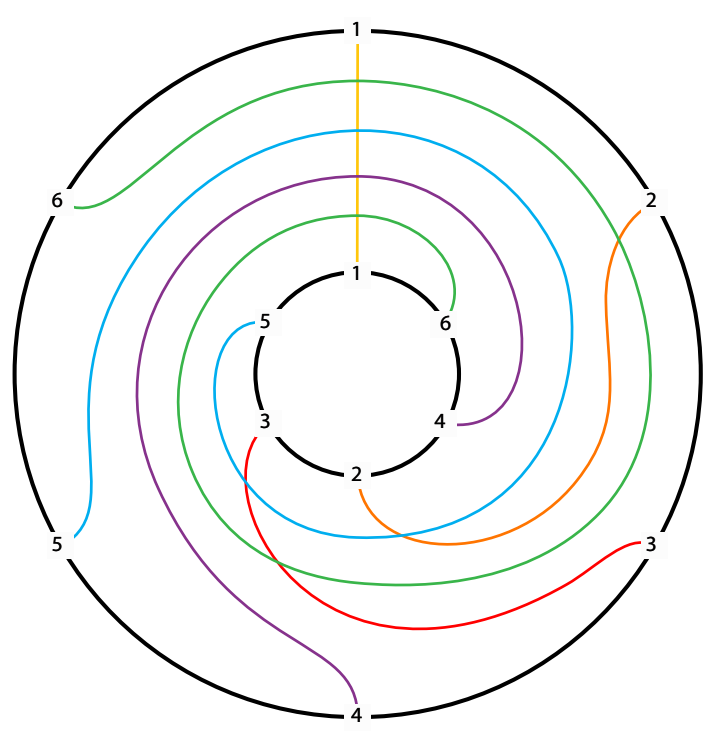

Figure 9: Projection of a circular line diagram representing the circular permutation $(1,6,4,2,3,5)$

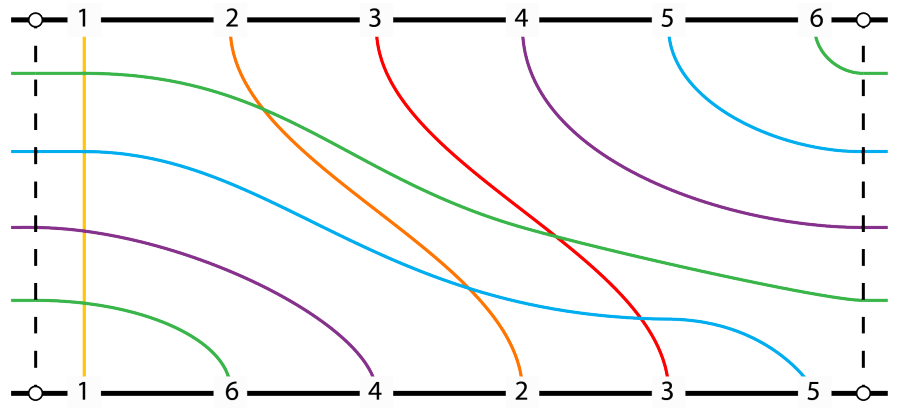

Figure 10: Unrolling of a circular line diagram representing the circular permutation $(1,6,4,2,3,5)$ 
We denote by $L_{i}(D)$ the line of $D$ that goes from $i$ at the top to $i$ at the bottom. We define $L_{i, j}(D)$ to be number of times that $L_{i}(D)$ and $L_{j}(D)$ cross each other.

For $i<j$, a crossing between the line $L_{i}(D)$ and $L_{j}(D)$ is called a legal crossing if

i) $i+1<j$,

ii) $L_{j}(D)$ crosses $L_{i}(D)$ from the right side of $L_{i}(D)$ to the left side of $L_{i}(D)$ when one follows $L_{i}(D)$ from the top to the bottom of the cylinder. See Figure 11.

We say that a circular line diagram is legal if all its crossings are legal. Note that a legal circular line diagram must satisfy $L_{i, i+1}(D)=0$ for all $1 \leqslant i<n$. The diagrams in Figures 9 and 10 are examples of legal diagrams.
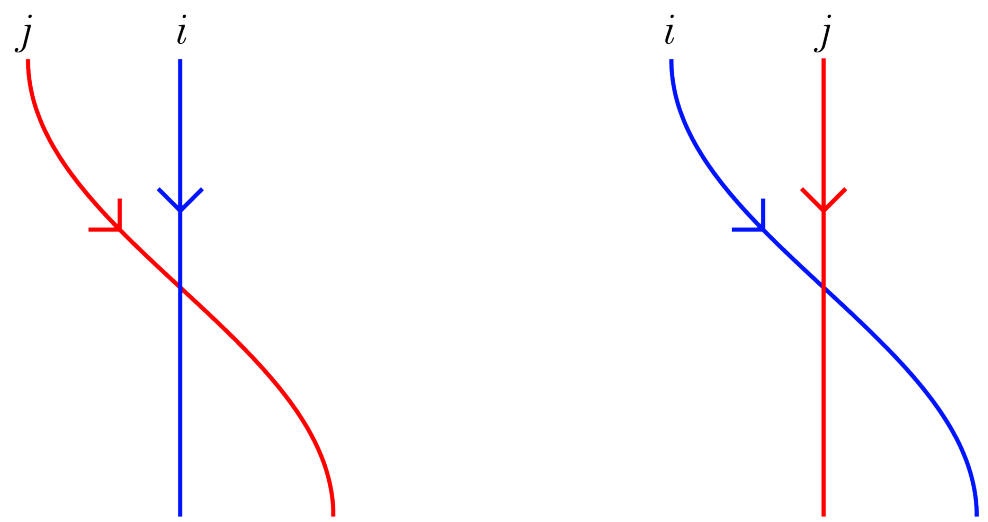

Figure 11: With $i+1<j$, the left crossing is legal while the right one is illegal.

We call trivial circular line diagram of $n$, the legal circular line diagram with no crossing. The trivial circular line diagram of $n$ represents the permutation $(1,2, \ldots, n)$.

Now, let $\sigma$ and $\tau$ be two permutations such that $(i j) \sigma(i j)=\tau$ for $i+1<j$ and $D_{\sigma}$ a legal circular line diagram representing $\sigma$. One can build a legal diagram representing $\tau$ by interchanging $i$ and $j$ at the bottom of $D_{\sigma}$. Because $i j$ is a large circular descent, it will only create a crossing between $L_{i}\left(D_{\sigma}\right)$ and $L_{j}\left(D_{\sigma}\right)$, which will be a legal crossing. Thus, this new diagram would be a legal circular line diagram representing $\tau$.

Therefore, by starting with the trivial circular line diagram, for any circular permutation $\sigma$, one can build a legal circular line diagram $D$ representing $\sigma$. With this construction, it is straightforward that $D$ has the property that $L_{i, j}(D)=v_{i j}$, where $v=V(\sigma)$. In fact, we will prove that this property holds for any legal diagrams. For example, if one counts the crossings of Figure 10, one will obtain the admitted vector of Figure 12. We will also see, throughout the next results a links between upward path in the poset of circular permutation and legal line diagrams.

Considering two lines $L_{i}(D)$ and $L_{j}(D)$ of a circular line diagram $D$ that are crossing each other, we define the triangle $i-j$ of $D$, denoted $T_{i, j}(D)$, to be the triangle whose vertices are: the last intersection of $L_{i}(D)$ and $L_{j}(D)$ and the points $i$ and $j$ on the bottom of the cylinder. 
1

0

0
1

1

0

0
1

1

1

0

$0 \quad 0$

Figure 12: Admitted vector associated to the circular permutation $(1,6,4,2,3,5)$

Lemma 46. Let $D$ be a legal circular line diagram. If two lines are crossing each other, $D$ has a triangle that does not intersect any other line than its boundary.

Proof. We say that a triangle is basic if at least one of its non-bottom edges does not cross any line. There always exist such triangles. Indeed, follow a line $L_{i}(D)$ and consider the triangle whose top vertex is the last crossing of $L_{i}(D)$. Order basic triangles by inclusion of their surfaces. Let $T$ be a minimal element of this poset. Then $T$ satisfies the conclusion of this lemma.

Theorem 47. Let $D$ be a non-trivial legal circular line diagram representing a circular permutation $\sigma$. There exists a large circular ascent $a_{h}$ in $\sigma$ such that we can create a legal diagram $D^{\prime}$ that represent $\left(a_{h}, a_{h+1}\right) \sigma\left(a_{h}, a_{h+1}\right)$ where $L_{k, l}\left(D^{\prime}\right)=L_{k, l}(D)$, for any pair $k, l$, except for $a_{h}, a_{h+1}$ where $L_{a_{h}, a_{h+1}}\left(D^{\prime}\right)=L_{a_{h}, a_{h+1}}(D)-1$.

Proof. By Lemma 46 we know that there exists in $D$ a pair $i, j$ such $T_{i, j}(D)$ is not crossed by any line. Therefore, $i$ is a large circular ascent of $\sigma$, with $j$ following $i$. By interchanging $i$ and $j$ on the bottom of the cylinder, we can untie the last crossing between $L_{i}(D)$ and $L_{j}(D)$ and keep the rest of the diagram unchanged. This proves the result.

Corollary 48. Let $\sigma$ be a circular permutation and $D$ a legal circular line diagram representing $\sigma$. Let $v=V(\sigma)$. Then $L_{i, j}(D)=v_{i, j}$ for any pair $i, j$.

Proof. It is easy to convince ourself that the only legal circular line diagram that represents $(1,2, \ldots, n)$ is the trivial circular line diagram, which has no crossing.

The rest follows from Theorem 47 and the poset of circular permutations.

We finish this section by presenting two algorithms. The first one takes a circular permutation $\sigma$ and returns a legal circular line diagram representing $\sigma$. Thus, we can deduce the admitted vector $V(\sigma)$.

The second one takes an admitted vector $v$ and returns a legal circular line diagram $D$ that has the property that $L_{i j}(D)=v_{i j}$. Thus, $D$ represent the circular permutation $V^{-1}(v)$. 
For the first algorithm: take a cylinder and mark the numbers 1 to $n$ on top in order and the circular permutation $\sigma$ at the bottom; preferably with 1 at the bottom align with 1 at the top. We draw a line linking both 1 . Now, for $j$ going from 2 to $n$, we draw a line going from $j$ at the top to $j$ at the bottom without crossing $j-1$ and by only doing crossing if necessary. Doing so, we are only doing legal crossing, and thus, building a legal circular line diagram. The algorithm ends when we have placed the $n$ lines.

For the second algorithm: take a cylinder and mark the numbers 1 to $n$ on top in order. Then mark the number 1 on the bottom line below the 1 from the top line. Draw a line from 1 at the top to 1 at the bottom.

For $j$ going from 2 to $n$, let $r_{j}=\left(a_{1}, \ldots, a_{j-1}\right)$ with $a_{i}=v_{i, j}$.

i) For $k$ going from 1 to $j-1$, if $a_{k} \neq 0$ make $L_{j}$ cross $L_{k}$ and $a_{k}$ becomes $a_{k}-1$.

ii) If $r_{j} \neq(0, \ldots, 0)$ redo step $\left.i\right)$, if $r_{j}=(0, \ldots, 0)$ then make $L_{j}$ drop to the bottom of the cylinder without crossing any line. Place $j$ where $L_{j}$ touches the bottom of the cylinder.

The algorithm ends when we have placed the $n$ lines.

Note that, if we look at admitted vectors with the coordinates placed in a pyramidal shape, this algorithm is running through each diagonal a certain number of time, as shown in Figure 13.

We give an example of the second algorithm in Figure 14. The corresponding circular permutation is $(1,5,3,2,4)$. The lines $L_{1}, L_{2}, L_{3}, L_{4}$ are directly drawn. We only detail the steps of the line $L_{5}$.

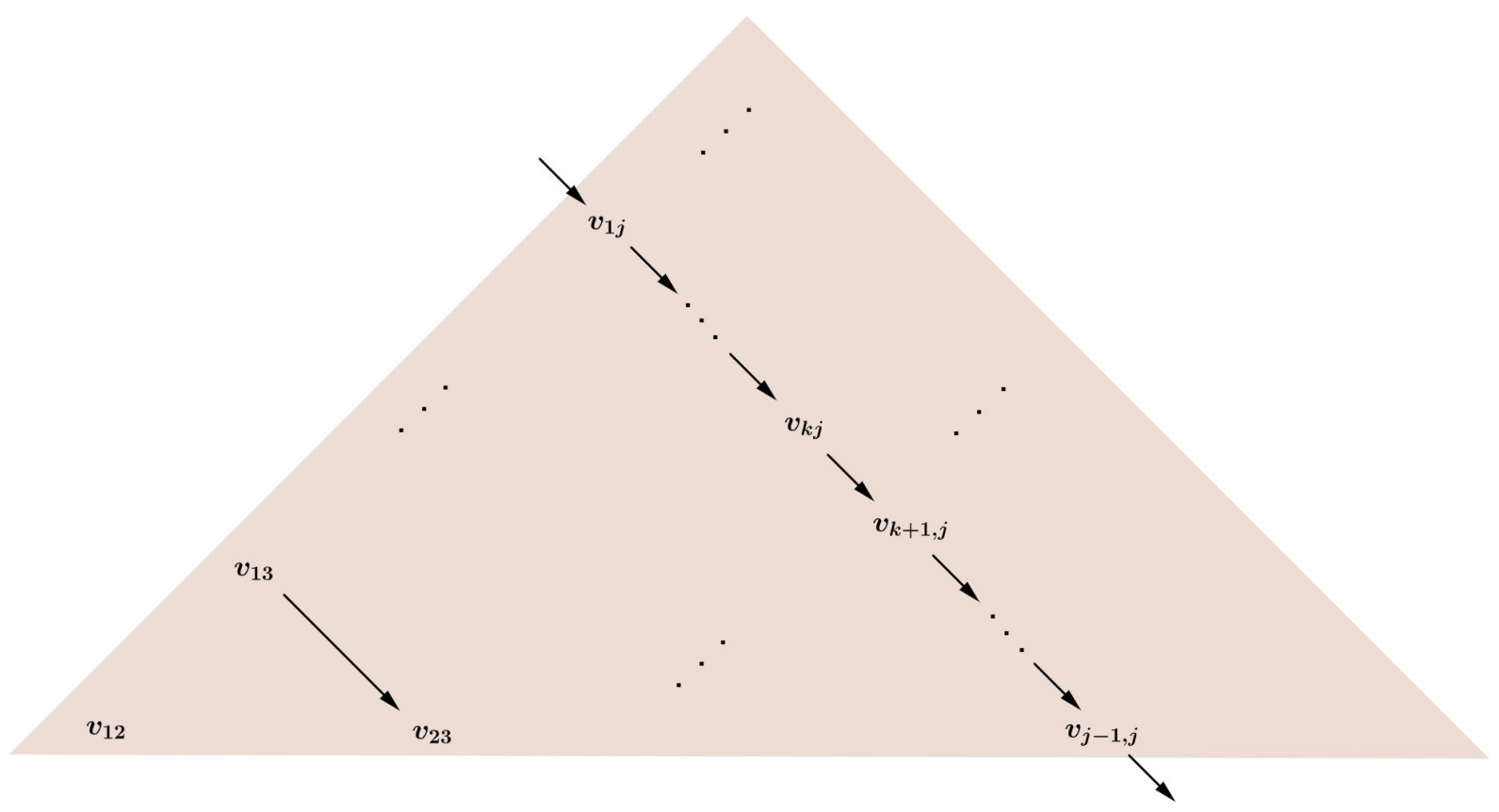

Figure 13: Reading direction of an admitted vector $v$ in the diagonal descent algorithm. 

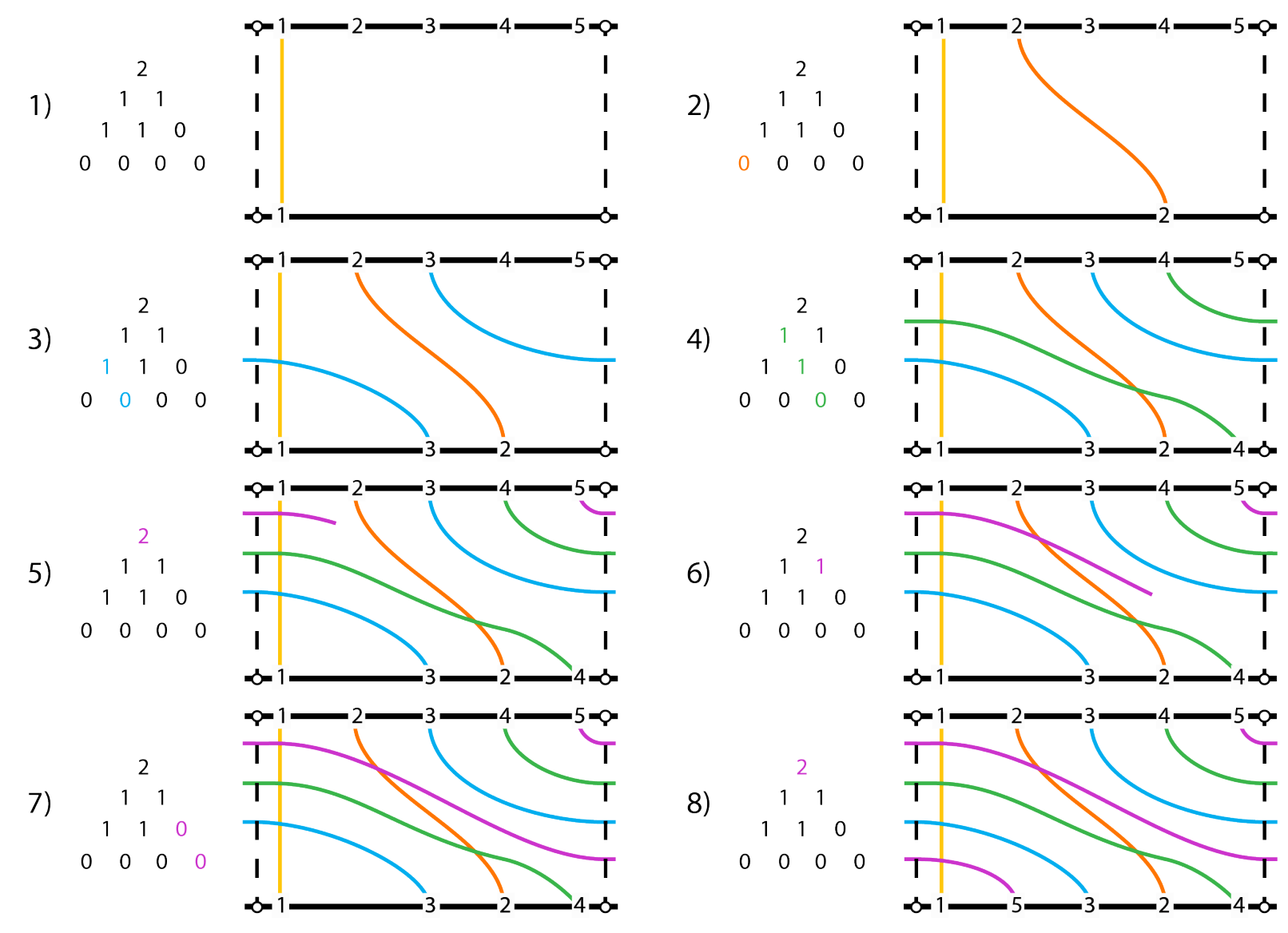

Figure 14: The diagonal descent algorithm.

\section{Further Questions}

First of all, we mention a link between our poset, and the poset in [9] when specialized to $A_{n}$.

Conjecture 49. The poset of circular permutations and the poset in [9] for the case $A_{n}$ are isomorphic.

We do not have a proof of this isomorphism, but the sizes and the mathematical context of both posets suggest that they are isomorphic.

In their article, Gashi et al. were able to give a closed formula for the rank generating function of their poset (see [9], Eq.(5.7)). Thus, if the isomorphism exists, it is also the rank generating function of our poset.

An interesting open problem, linked to the work of Gashi et al., would be to look at other affine Coxeter groups and see if some nice combinatorial objects arise.

In 1927, K. Reidemeister introduced 3 moves in knot theory [14]: the Reidemeister moves. One can observe that Reidemeister move of type III works well with legal circular line diagrams in the following way: one can pass from a legal diagram to another that represents the same permutation using such move. 

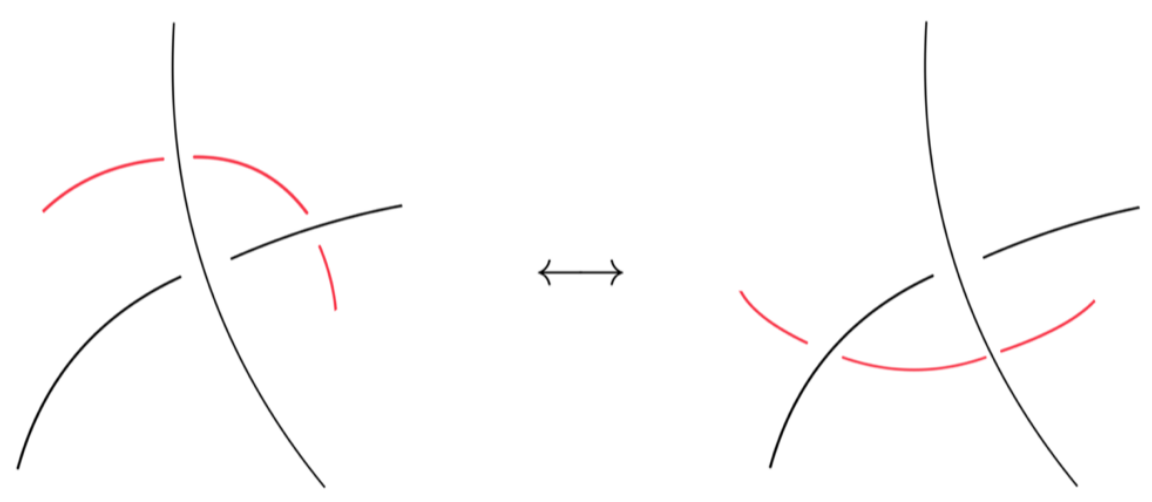

Figure 15: Reidemeister move of type III.

We think that more might occur:

Conjecture 50. Let $\sigma$ be a circular permutation and let $D_{\sigma}, D_{\sigma}^{\prime}$ be two legal circular line diagrams representing $\sigma$. Then we can obtain $D_{\sigma}^{\prime}$ from $D_{\sigma}$ using only Reidemeister moves of type III.

From this conjecture comes other questions.

Question 51. In Conjecture 50, what is the number of Reidemeister moves of type III needed to obtain $D_{\sigma}^{\prime}$ from $D_{\sigma}$ ?

Question 52. What is the number of legal diagrams representing the same circular permutation?

In order to answer that last question, we think that we have to put an order on the crossings in a circular line diagram by taking their order of appearance along the cylinder from top to bottom in the same way as pseudo-line arrangements (see [2] or [10]). With this order of crossings in mind, we can define the following equivalence relation: two diagrams are equivalent if they represent the same permutation and have the same order of appearance of the crossings. We then have the following conjecture:

Conjecture 53. The number of circular line diagrams representing a permutation $\sigma$ is exactly the number of paths in the poset of circular permutations from the permutation $(1,2, \ldots, n)$ to $\sigma$.

As Berenstein et al. explain in Section 2 of [2], we know that pseudo-line arrangements are in bijection with reduced expression of elements in $S_{n}$.

Question 54. Are circular line diagrams in bijection with reduced expression of circular permutations? 
Finally, an interesting object of finite poset are the maximal chains, more particularly, the number of these chains. We were able to calculate the number of maximal chains for $n$ up to 9 . This sequence does not appear in OEIS yet.

\begin{tabular}{|r|r|}
\hline$n$ & number of maximal chains \\
\hline 2 & 1 \\
3 & 1 \\
4 & 2 \\
5 & 60 \\
6 & 279180 \\
7 & 709628135986073648478240 \\
8 & 2563983592380 \\
9 & 105883974421582156430080943763802159752672 \\
\hline
\end{tabular}

\section{Acknowledgements}

The authors thank Nicolas England, Christophe Hohlweg, Hugh Thomas and Richard Stanley for useful discussions and mails. We also thank the referee for his/her thorough reading of our article and for many useful suggestions. Finally we thank Darij Grinberg, Travis Schedler and David Speyer; they brought to our attention respectively [1] (see Remark 6) and [9].

\section{References}

[1] Art of Problem Solving. USAMO 2010 Problem 2. https://artofproblemsolving. com/wiki/index.php/2010_USAMO_Problems/Problem_2.

[2] Arkady Berenstein, Sergey Fomin, and Andrei Zelevinsky. Parametrizations of Canonical Bases and Totally Positive Matrices. Advances in Mathematics, 122(1):49149, 1996.

[3] Anders Björner and Francesco Brenti. Affine Permutations of Type A. Electronic Journal of Combinatodics, 3(2), \#R18, 1996.

[4] Anders Björner and Francesco Brenti. Combinatorics of Coxeter Groups, volume 231 of Graduate Texts in Mathematics. Springer, New York, 2005.

[5] Nathan Chapelier-Laget. Shi Variety Corresponding to an Affine Weyl Group. arXiv: 2010.04310.

[6] Nathan Chapelier-Laget. A Symmetric Group Action on the Irreducible Components of the Shi Variety Associated to $W\left(\widetilde{A}_{n}\right)$. arXiv:2010.05602.

[7] Henrik Erikson and Kimmo Erikson. Affine Weyl Groups as Infinite Permutations. Electronic Journal of Combinatorics, 5, \#R18, 1998.

[8] Sergey Fomin and Zelevinsky Andrei. Cluster Algebras I: Foundations. J. Amer. Math. Soc., 15(2), 2002. 
[9] Qëndrim R. Gashi, Travis Schedler, and David E. Speyer. Looping of the Numbers Game and the Alcoved Hypercube. Journal of Combinatorial Theory, Series A, 119(3):713-730, 2012.

[10] Christian Kassel, Alain Lascoux, and Christophe Reutenauer. Factorizations in Schubert Cells. 2000.

[11] Witold Kraśkiewicz. Reduced Decompositions in Weyl Groups. European Journal of Combinatorics, 16(3):293-313, 1995.

[12] T. Kyle Petersen. Eulerian Numbers. Birkhaüser/Springer, 2015.

[13] Nathan Reading and David E Speyer. Sortable Elements in Infinite Coxeter Groups. Transactions of the American Mathematical Society, 363:699-761, 2011.

[14] Kurt Reidemeister. Elementare Begründung der Knotentheorie. In Abhandlungen aus dem Mathematischen Seminar der Universität Hamburg, volume 5, pages 24-32. Springer, 1927.

[15] Bruce Sagan. The Symmetric Group. Springer, 2000.

[16] Jian Yi Shi. On Two Presentations of the Affine Weyl Groups of Classical Types. Journal of Algebra, 221:360-382, 1999.

[17] Richard P. Stanley. Enumerative Combinatorics. Wadsworth and Brooks/Cole, 1, 1986. 